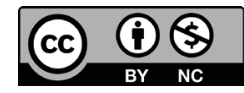

\title{
LA FORMA DI GOVERNO IN ITALIA DOPO IL 1993: MUTAMENTI E NUOVI ORIENTAMENTI
}

\author{
THE FORM OF GOVERNMENT IN ITALY AFTER \\ 1993: CHANGES AND NEW TRENDS \\ A FORMA DE GOVERNO NA ITÁLIA APÓS 1993: \\ MUDANÇAS E NOVAS ORIENTAÇÕES
}

\author{
Giorgio Sobrino
}

\section{RIASSUNTO}

Il lavoro descrive i mutamenti intervenuti nella forma di governo in Italia, ed i nuovi orientamenti emersi al riguardo, nel periodo successivo al 1993. Questo anno, nel quale si svolse il referendum popolare che determinò l'abrogazione della legge elettorale proporzionale che aveva regolato lo svolgimento delle elezioni in Italia dagli albori della Repubblica, segna infatti uno "spartiacque" nelle ricostruzioni della dottrina sulla forma di governo. Esso diede avvio ad una lunga fase di transizione della forma di governo italiana verso un assetto diverso da quello dei decenni precedenti, e a connotazione maggioritaria. Il lavoro, inoltre, evidenzia che a partire dal 2013/2014 si è aperta una fase ulteriore della forma di governo in Italia (oggi non ancora facilmente classificabile), per effetto del cambiamento del quadro politico determinato dall'irruzione del MoVimento 5 Stelle e, dall'altro lato, delle sentenze della Corte Costituzionale n. 1/2014 e n. 35/2017, che hanno dichiarato illegittime le ultime due leggi elettorali di impronta maggioritaria.

Parole-chiave: Sistema politico italiano. Forma di governo italiana. Legge elettorale. Parlamento. Governo. 


\section{ABSTRACT}

The paper describes the changes of the form of government in Italy, and the new trends that have occurred in this regard, in the period after 1993. This year, in which took place the popular referendum that repealed the proportional electoral law that had ruled the elections in Italy since the first years of the Republic, represents a "watersched" in the reconstruction of the doctrine on the form of government. It initiated a long period of transition of the Italian form of government towards a structure different from the previous one, with a majority connotation. The paper also highlights that starting from 2013/2014 a further phase of the form of government in Italy has opened (today not yet easily classified), due to the change in the political system determined by the irruption of the "MoVimento 5 Stelle" and, on the other hand, to the judgments of the Constitutional Court $n^{\circ} 1 / 2014$ and $n^{\circ} 35 / 2017$, which declared the last two majority electoral laws illegitimate.

Keywords: Italian political system. Italian form of government. Electoral rule. Parliament. Cabinet.

\section{RESUMO}

O presente artigo descreve as mudanças na forma de governo na Itália, e as novas tendências que ocorreram nesse sentido, no período posterior a 1993. Este ano em que ocorreu o referendo popular que revogou a lei eleitoral proporcional que havia regulamentado as eleições na Itália desde os primeiros anos da República - representa um "divisor de águas" na reconstrução da doutrina sobre a forma de governo. Iniciou-se um longo período de transição da forma de governo italiana para uma estrutura diferente à anterior, com uma conotação majoritária. Este artigo também destaca que, a partir de 2013/2014, iniciou-se uma nova fase da forma de governo na Itália (hoje ainda não facilmente classificável), devido à mudança no sistema político determinada pela irrupção do "MoVimento 5 Stelle" e, por outro lado, aos acórdãos do Tribunal Constitucional n ${ }^{\circ} 1 / 2014$ e $n$ ○35/2017, que declararam ilegítimas as duas últimas leis eleitorais majoritárias.

Palavras-chave: Sistema político italiano. Forma de governo italiana. Lei eleitoral. Parlamento. Governo. 


\section{CONSIDERAZIONI INTRODUTTIVE: I MUTAMENTI DELLA FORMA DI GOVERNO ITALIANA DAL 1993; LA NOZIONE DI FORMA DI GOVERNO NELLA DOTTRINA ITALIANA ED IL METODO D’INDAGINE QUI SEGUITO}

In questa sede si intende proporre una riflessione sui mutamenti e sui nuovi orientamenti - che hanno interessato la forma di governo in Italia a partire, almeno, dal 1993.

L'evoluzione della forma di governo rappresenta, invero, un elemento centrale della vicenda costituzionale italiana nel periodo dal 1993 ad oggi, imprescindibile per chi intenda analizzare da un punto di vista costituzionalistico questo periodo della storia della Repubblica, assai controverso. Il 1993 - e più precisamente, come si vedrà meglio oltre, il 18 aprile 1993, data di svolgimento del referendum popolare che determinò l'abrogazione (con una larghissima maggioranza) della legge elettorale di tipo proporzionale che aveva regolato lo svolgimento delle elezioni in Italia fin dagli albori della Repubblica - segna, a questo proposito, una data "spartiacque" nelle ricostruzioni della dottrina: il crollo del sistema dei partiti tradizionali e la comparsa sulla scena politica di soggetti del tutto nuovi, "a vocazione maggioritaria" (in primo luogo Forza Italia di Silvio Berlusconi ${ }^{1}$ ), diede avvio $^{2}$ ad una lunga fase di transizione, appunto, della forma di governo italiana verso un assetto diverso da quello dei decenni precedenti, e a connotazione (parlamentare) maggioritaria ${ }^{3}$.

Tale periodo di transizione - come pure si vedrà nel corso di questo scritto - ha trovato un ulteriore punto di snodo a distanza di vent'anni, con le elezioni politiche del 2013 e l'ingresso in Parlamento (non meno dirompente quanto a caratteristiche ed effetti) del MoVimento 5 Stelle, fondato da Beppe Grillo: ciò ha determinato la fine del "bipolarismo", basato sulla competizione tra una coalizione di partiti di ispirazione conservatrice (di "centrodestra", guidata costantemente da Silvio Berlusconi) ed una di partiti di ispirazione progressista (di "centrosinistra", guidata invece nel tempo da leaders diversi) ${ }^{4}$. Questa fine è stata successivamente "certificata" sul piano normativo dalle sentenze n. 1/2014 e n. 35/2017 della Corte Costituzionale, che hanno dichiarato costituzionalmente illegittime (ed espunto, perciò, dall'ordinamento) le modifiche apportate alla legge 
elettorale nel 2005 e nel 2015, entrambe volte a consolidare il sistema maggioritario, e dalla conseguente riforma elettorale del $2017^{5}$, con cui il legislatore ha (re-)introdotto un sistema elettorale "misto" con una quota prevalente di seggi assegnata, tuttavia, con metodo proporzionale ${ }^{6}$.

Da ultimo, le elezioni politiche del 4 marzo 2018 e gli eventi successivi hanno aperto una fase politico- istituzionale particolarmente fluida e complessa, nella quale la forma di governo parlamentare italiana sembra esibire delle caratteristiche proprie del periodo antecedente al 1993 - in primo luogo il «multipartitismo esasperato» ${ }^{7}$ e l'estrema instabilità dei Governi $^{8}$-, sollevando inoltre dei problemi in parte nuovi (e a loro volta rilevanti agli effetti della connotazione della forma di governo stessa), su cui oggi i costituzionalisti si stanno interrogando 9 .

L'analisi della forma di governo e delle sue dinamiche evolutive rappresenta, dunque, un punto d'osservazione per certi versi privilegiato della fase di "transizione" che ha vissuto - e sta vivendo tuttora - il sistema costituzionale italiano ${ }^{10}$, nel quadro peraltro di fenomeni caratterizzati ormai da una rilevante dimensione sovranazionale. In tale contesto, il presente contributo si propone di descrivere i mutamenti intervenuti nella forma di governo in Italia, ed i nuovi orientamenti emersi al riguardo, incentrando l'analisi - per le ragioni sopra indicate - sul periodo successivo al 1993 (salvi ovviamente i richiami all'esperienza del periodo precedente che si renderanno necessari, in chiave di confronto).

Ai fini dell'analisi occorre, preliminarmente, dare conto della nozione di forma di governo invalsa nella dottrina costituzionalistica italiana ed assunta perciò a fondamento della trattazione - e, dall'altro lato, del metodo seguito nella presente indagine.

Quanto alla nozione di forma di governo, va richiamata la definizione elaborata negli anni Settanta dello scorso secolo da Mortati ed Elia ${ }^{11}$ (sulla quale si registra tuttora un consenso larghissimo): modo in cui sono distribuite le funzioni politiche ${ }^{12}$ tra i diversi organi dello Stato e (in secondo luogo) i rapporti che intercorrono tra di essi, in base alle norme dell'ordinamento considerato (in primis quelle costituzionali) ${ }^{13}$.

A partire da tale nozione generale, la dottrina italiana ha proposto diverse classificazioni delle forme di governo - e dunque "tipi" di forme di governo, da utilizzare come modelli per analizzare le forme 
di governo storiche (compresa quella italiana nei differenti periodi) -, valorizzando l'uno o l'altro elemento discretivo. In particolare, sempre Elia $^{14}$ - basandosi sul criterio del rapporto tra il potere legislativo e l'esecutivo e (correlativamente) della modalità di investitura dell'esecutivo - ha distinto le forme di governo in quattro tipi: quella parlamentare; quella presidenziale ${ }^{15}$; quella semipresidenziale $(0$, più precisamente, «a componenti presidenziali e parlamentari» ${ }^{16}$ ); quella direttoriale. La forma di governo parlamentare, a sua volta, è stata suddivisa da Elia in tre diverse «varianti» (riscontrabili in concreto, secondo questo Autore, negli ordinamenti che la prevedono): il parlamentarismo «a bipartitismo rigido»; quello «a multipartitismo temperato»; infine, quello «a multipartitismo esasperato» ${ }^{17}$. Questa ulteriore distinzione è conseguenza del rilievo primario che Elia attribuiva al sistema dei partiti ai fini della conformazione delle forme di governo (profilo su cui si tornerà tra breve). Un altro studioso, Pinelli ${ }^{18}$, più di recente - riprendendo una distinzione del già citato Mortati ${ }^{19}$ e utilizzando il criterio discretivo dell'organo dotato di investitura popolare diretta - ha invece prospettato una bipartizione delle forme di governo: quelle «moniste», caratterizzate dall'elezione popolare soltanto del parlamento (forma di governo parlamentare e direttoriale); quelle «dualiste», dall'altro lato, caratterizzate dall'elezione popolare sia del parlamento che del presidente (forma di governo presidenziale e quella - che Pinelli definisce «parlamentare» come quella «monista» di cui sopra - in cui pure il presidente è eletto dal popolo, ma si assiste ad un rapporto di forza "invertito" tra quest'organo ed il circuito parlamentogoverno, rispetto al presidenzialismo "puro" ${ }^{20}$ ).

In relazione a queste classificazioni generali, va osservato che la forma di governo italiana nella sua dimensione storico- effettiva si colloca, secondo Elia, fino al 1993 nella categoria (o «variante») del parlamentarismo «a multipartitismo esasperato». Nel periodo «successivo al referendum del 1993» (già ricordato), invece, «la nuova legislazione elettorale italiana ha incentivato un bipolarismo di investitura con risultati non trascurabili rispetto al periodo più decadente della c.d. prima repubblica» ${ }^{21}$ : si pone allora il problema di comprendere se le vicende politico- costituzionali intervenute da quella data fino ai nostri giorni abbiano determinato o meno il passaggio della forma di governo ad un'altra «variante» di 
parlamentarismo $^{22}$ (in proposito v. oltre, in particolare $\S 4$ ). Rispetto invece alla tassonomia proposta da Pinelli, è pacifica la riconducibilità della forma di governo italiana - sia prima che dopo lo snodo del 1993 - alle forme di governo «moniste», caratterizzate, come si è visto, dall'elezione popolare soltanto del parlamento ${ }^{23}$.

Occorre peraltro ricordare, in questa sede introduttiva - richiamando un'altra autorevole dottrina ${ }^{24}$-, i limiti insiti nelle classificazioni generali, come quelle sopra esposte. Esse infatti, in quanto basate sulla "tipizzazione" di elementi presenti in realtà politico- costituzionali spesso molto diverse tra loro, e costrette perciò a considerare «variabili» concrete numerose ed eterogenee, scontano spesso - nel passaggio dall'analisi astratta all'esame effettivo dei sistemi politici -, un'eccessiva rigidità, che ne compromette l'utilità pratica ${ }^{25}$. Ciò emerge proprio nell'analisi dell'esperienza italiana, che nel periodo dopo il 1993 è caratterizzata da vicende contraddittorie e, dunque, da un'elevata fluidità: è difficoltoso e non univoco il suo inquadramento in una delle categorie astratte innanzi ricordate (sul punto v. ancora oltre, in particolare § 4). Secondo Luciani, in particolare, ad una ricostruzione della forma di governo basata su modelli e classificazioni astratte è preferibile l'analisi dell'esperienza delle forme di governo storiche (il parlamentarismo inglese, il semipresidenzialismo della $\mathrm{V}$ Repubblica francese, ecc.), per poi ricavare da esse, successivamente, alcuni «elementi» rilevanti e mettere a confronto la relativa disciplina nei diversi ordinamenti positivi ${ }^{26}$.

Venendo ora (in stretta correlazione a quest'ultima notazione) all'aspetto del metodo di analisi della forma di governo - e quindi dell'indagine qui svolta -, la dottrina italiana ha seguito approcci diversi a seconda della posizione assunta su questo problema teorico: se la natura e la conformazione effettiva di una forma di governo dipendano dalle norme giuridiche di quel determinato ordinamento oppure da altri elementi $e$ fattori, come per esempio i «fatti politici» ed i «comportamenti» pratici dei diversi attori in gioco (organi dello Stato, ma anche altri soggetti). In altri termini (e più precisamente): la dottrina si è interrogata su quale incidenza abbiano le norme giuridiche - quelle costituzionali in primo luogo - ed i «fatti politici» - od altri eventuali fattori - sulla connotazione della forma di governo di un particolare ordinamento costituzionale. 
Nello specifico, da una parte, Elia ${ }^{27}$ (seguito dalla maggior parte degli studiosi ${ }^{28}$ ) ha proposto un approccio "realistico" - o "fattuale" all'indagine sulla forma di governo, che si caratterizza per attribuire un rilievo primario ai fatti concreti (le prassi, i precedenti, le «regolarità» ricorrenti nel funzionamento di un determinato sistema istituzionale) ed al «contesto politico» rispetto alle norme che regolano in astratto i rapporti tra gli organi costituzionali. I «fatti» - così intesi - secondo Elia sono, emblematicamente, «situazioni condizionanti» la forma di governo ${ }^{29}$; tra queste la più importante è il sistema dei partiti: «le forme di governo dello stato democratico non possono più essere classificate né studiate, anche dal punto di vista giuridico, prescindendo dal "sistema dei partiti" ${ }^{30}$. Dall'altra parte, Luciani ${ }^{31}$, in particolare - muovendo dalla tesi per cui la categoria della «forma di governo» si identifica esclusivamente con la «struttura» dei rapporti tra i poteri e gli organi costituzionali ${ }^{32}$, mentre il funzionamento del sistema dei partiti e le prassi politico- costituzionali ne rappresentano il mero «contenuto materiale» -, ha prospettato un metodo di analisi in base al quale: -1 ) le regole formali e la prassi (cioè la «struttura» ed il «contenuto» di cui si è detto) devono essere tenute ben distinte nell'indagine di una determinata forma di governo, attribuendosi un rilievo definitorio e "qualificante" la forma di governo stessa ${ }^{33}$ solo alle prime ${ }^{34}$; -2) tali regole e prassi (o «norme» e «fatti politici») devono essere intesi come «variabili reciprocamente dipendenti», che concorrono a determinare (non già le «forme di governo» in sé, ma piuttosto) le «concrete esperienze giuridico- politiche». «L'effettivo funzionamento di un sistema politico - istituzionale dipende da una serie di fattori materiali non meno che dalle regole formali del suo funzionamento; nondimeno, così come i fattori materiali condizionano l'esito applicativo delle regole formali, quelle regole formali costituiscono le condizioni di possibilità delle concrete strategie degli attori politici del sistema ... Per la comprensione dell'oggetto nella sua unitarietà (e cioè delle concrete, storiche, esperienze giuridico - politiche) [è] indispensabile il riferimento tanto alla struttura quanto al funzionamento» 35 .

Tra queste diverse impostazioni metodologiche, nel presente lavoro ci si muoverà - per così dire - su un piano "intermedio", che intende valorizzare quello che sembra il punto di contatto fra esse ${ }^{36}$. Le due 
impostazioni, infatti - al di là delle loro diverse premesse definitorie e, appunto, di metodo -, paiono convergere sull'idea per cui ogni «forma di governo storica» è il risultato dell'interazione tra le norme giuridiche ed i fatti (o gli «elementi», o «fattori») materiali di quel determinato sistema politico, e viceversa. Questa interazione ha delle caratteristiche e delle "direzioni" diverse di caso in caso (a seconda, cioè, dell'ordinamento costituzionale considerato), e di periodo storico in periodo storico (all'interno di uno stesso ordinamento costituzionale). Dunque l'esame della forma di governo italiana del periodo dopo il 1993 che seguirà si articolerà in due principali passaggi:

-1) in primo luogo ( $\S \S 2$ - 4), nell'indagine delle caratteristiche e dell'evoluzione concreta, durante questo arco temporale, di determinati "elementi materiali" del sistema politico- istituzionale italiano (quelli oggetto di esame più frequente da parte della dottrina costituzionalistica): -a) il sistema dei partiti; -b) la formazione dei governi ed il rapporto di fiducia parlamento- governo (sua instaurazione e vicende successive); -c) la crisi dei governi e la prassi dello scioglimento anticipato delle camere; -d) il ruolo del governo ed il suo rapporto, in particolare, con gli organi di garanzia. Come suggerisce l'insegnamento di Elia, tali elementi - e le tendenze generali della loro evoluzione, che saranno oggetto di valutazione specifica (nel § 4) - risultano particolarmente importanti per la conformazione (soprattutto) di una forma di governo parlamentare ${ }^{37}$, come quella italiana;

-2) in secondo luogo ( $\S \S 5$ - 6), nell'analisi di come le disposizioni costituzionali sulla forma di governo in Italia (artt. 55 e seguenti della Carta, ed in particolare artt. 92 e seguenti) sono state interpretate dagli attori politici ed istituzionali sempre dopo il 1993; e, correlativamente, del rapporto che si è determinato tra queste regole formali ed $\mathrm{i}$ «fatti politici» più rilevanti di tale periodo. Come sottolinea, questa volta, l'impostazione di Luciani, tale (specifico) rapporto è ciò che determina in concreto - come si è visto sopra - la forma di governo ${ }^{38}$ oggetto di attenzione.

In sostanza (e per tradurre in modo più concreto i due passaggi analitici appena esposti): si cercherà di comprendere se gli eventi - e gli indubbi mutamenti - che hanno interessato il sistema politico e la prassi degli organi costituzionali in Italia dopo la data- "spartiacque" del 1993 
abbiano comportato un mutamento della forma di governo, intesa come «struttura» formale dei rapporti tra gli organi costituzionali; e, se sì, in che senso. In particolare, occorrerà chiarire se (e anche qui, in quali termini) le regole stabilite dalla Costituzione italiana sulla forma di governo siano state condizionate, nella loro interpretazione ed applicazione, dalla - o abbiano a loro volta condizionato la - evoluzione del sistema politico di questi anni; e ciò anche con riferimento ai numerosi progetti di riforma di tali norme che, come pure si dirà (nel § 6), sono stati presentati dalle diverse forze politiche lungo questi venticinque anni, che si fondano su un sostanziale giudizio di "obsolescenza" del dettato costituzionale originario a fronte - appunto - del mutamento del quadro politico nel frattempo intervenuto.

\section{GLI ELEMENTI MATERIALI DELLA FORMA DI GOVERNO ITALIANA NEL PERIODO DOPO IL 1993: CARATTERISTICHE ED EVOLUZIONE}

\section{IL SISTEMA DEI PARTITI (DINAMICHE SUCCESSIVE ALLO SVOLGIMENTO DELLE ELEZIONI)}

Iniziando allora l'analisi dal (l'“elemento materiale"-) sistema dei partiti, occorre premettere che esso viene preso qui in considerazione soltanto negli aspetti più direttamente legati alla forma di governo: le modalità di strutturazione e l'assetto dei partiti dopo lo svolgimento delle elezioni politiche; il comportamento degli stessi all'interno delle coalizioni; correlativamente, l'apporto ed il "peso" dei partiti e delle coalizioni nell'assunzione delle decisioni politiche di maggiore rilievo ${ }^{39}$.

Ciò precisato, si osserva che questo elemento, nel periodo dopo il 1993, presenta due principali tendenze evolutive, senza dubbio innovative rispetto alla lunga fase di storia repubblicana precedente (dal $1948 \mathrm{al}$, appunto, 1992/93) ${ }^{40}$. In primo luogo - e con riferimento all'assetto del sistema partitico come tale -, la trasformazione radicale dovuta alla scomparsa (repentina e, per certi aspetti, drammatica ${ }^{41}$ ) dei principali partiti che avevano caratterizzato la scena politica italiana dalla fine 
della seconda guerra mondiale e, dall'altro lato, alla nascita di soggetti politici nuovi, che tentano di "occupare" tale scena politica e di raccogliere l'eredità ed il consenso elettorale dei primi. Dal 1948 fino agli anni Ottanta il sistema politico italiano era stato caratterizzato, principalmente, dal «multipartitismo esasperato» ${ }^{42}$ - presenza di molti partiti, alcuni dei quali con un consenso elettorale assai basso, agevolati da una legge elettorale di tipo rigidamente proporzionale - e dalla c.d. «conventio ad excludendum» nei confronti del Partito comunista, in base alla quale esso (per ragioni legate all'ordine delle relazioni internazionali durante il periodo della «guerra fredda») non poteva di fatto accedere al governo ${ }^{43}$. Tra il 1991 ed il 1993, per effetto delle inchieste giudiziarie contro la corruzione politica note come «Tangentopoli» e - dall'altro lato - del mutamento del contesto internazionale seguito al crollo dell'Unione Sovietica, si sciolgono uno dopo l'altro il Partito comunista italiano, il Partito socialista e la Democrazia cristiana (vero "baricentro" della vita politica in Italia per oltre quarant'anni); il vuoto lasciato, in particolare, dagli ultimi due partiti apre la strada a soggetti politici nuovi, come Forza Italia (fondata da Silvio Berlusconi dopo la sua «discesa in campo» del gennaio del $1994^{44}$ ), la Lega nord (nata solo pochi anni prima), il Partito popolare e Alleanza nazionale, erede del Movimento sociale italiano (che, nel corso della «Prima Repubblica», era stato a sua volta espressione dell'eredità ideologica del fascismo). Inoltre, l'abolizione della legge elettorale proporzionale derivante dal già ricordato referendum popolare del 18 aprile 1993, insieme ancora al mutamento del contesto geopolitico internazionale, pongono le condizioni per una semplificazione del quadro politico ${ }^{45} \mathrm{e}$ per l'apertura dell'area di governo ai partiti della sinistra, eredi del Pci (Pds, Rifondazione comunista e poi soprattutto il Partito democratico) ${ }^{46}$. Un'ulteriore significativa trasformazione - o, quantomeno, evoluzione del sistema partitico avviene poi a partire dal 2013, con l'irruzione sulla scena politica nazionale del MoVimento 5 Stelle fondato da Beppe Grillo (i cui esponenti vengono eletti in Parlamento per la prima volta, appunto, nel 2013) e la contestuale crisi dei due principali partiti della «Seconda Repubblica», Forza Italia da una parte ed il Partito democratico dall'altra (di cui oggi si sta assistendo alle conseguenze politiche). 
In secondo luogo - e sempre in correlazione all'intervenuto cambiamento del sistema elettorale - emerge la tendenza, altrettanto inedita nel contesto politico italiano, all'aggregazione dei partiti in coalizioni, che si formano prima delle elezioni e si propongono di sostenere un determinato governo per tutta la durata della legislatura che seguirà. Questa tendenza, nel periodo temporale qui considerato, emerge in modo inizialmente faticoso e accidentato; in seguito (all'incirca tra la seconda metà degli anni Novanta ed il primo decennio degli anni Duemila ${ }^{47}$ ) si afferma con maggiore stabilità e grado di condivisione nel sistema politico, fino a caratterizzare la forma di governo come oggettivamente diversa rispetto a quella dei decenni precedenti ${ }^{48}$; da ultimo, dopo la fine del "bipolarismo" dovuta all'affermarsi del MoVimento 5 Stelle ed il superamento del sistema elettorale maggioritario ad opera delle sentenze della Corte Costituzionale del 2014 e del $2017^{49}$ e della legge n. 165/2017, sembra di nuovo "mostrare la corda" ed essere messa in discussione dagli attori politici ${ }^{50}$. Peraltro, anche nella fase di più elevata propensione dei partiti a formare coalizioni "di legislatura", i loro comportamenti dopo lo svolgimento delle elezioni mostrano una prevalente instabilità: tutte le legislature dal 2001, in particolare, registrano (al pari delle precedenti) le dimissioni anticipate del governo nominato dopo le elezioni, e in due casi (legislature 2006/2008 e 2008/2013) ciò determina la fine della maggioranza politica scaturita dalle elezioni (rispettivamente, di centrosinistra e di centrodestra) ${ }^{51}$.

L'analisi dello svolgimento delle legislature dal 1993 in poi evidenzia, inoltre - nella prospettiva dell'indagine sulla forma di governo -, altri fenomeni meritevoli di sottolineatura. Da un lato, la "fluidità" dell'assetto dei partiti ${ }^{52}$ e (in stretta correlazione) la mobilità degli stessi rispetto alle coalizioni politiche che si vanno via via formando: la tendenza del sistema politico italiano a conformarsi secondo uno schema "bipolare", simile a quello dei parlamentarismi maggioritari ${ }^{53}$, risulta ostacolata - se non proprio impedita, se si guarda l'esito finale del processo considerato dalla tendenza contraria dei singoli partiti ${ }^{54}$ ad uscire dalle - o ad entrare nelle - coalizioni durante le legislature. Dall'altro lato, una "non fisiologica" (o, se si preferisce, "patologica") instabilità delle relazioni tra i partiti politici, e tra questi e gli organi costituzionali; in particolare il governo ${ }^{55}$. 
In definitiva, il sistema dei partiti, nel periodo dal 1993 ad oggi, mostra delle tendenze evolutive non univoche e talvolta anche fortemente contrastanti.

\section{LA FORMAZIONE DEI GOVERNI ED IL RAPPORTO DI FIDUCIA PARLAMENTO- GOVERNO (INSTAURAZIONE E VICENDE SUCCESSIVE)}

Passando ad esaminare le modalità di formazione dei governi $\mathrm{e}$ le (ad esse correlate) dinamiche del rapporto di fiducia tra questi ed il parlamento ${ }^{56}$, nel periodo temporale in considerazione emergono tre linee di mutamento rilevanti agli effetti della definizione della forma di governo.

In primo luogo, si registra la tendenza alla semplificazione delle procedure volte alla formazione dei governi ed all'instaurazione del rapporto di fiducia, ai sensi dell'art. 94 Cost. ${ }^{57}$. Dopo il 1993, e soprattutto nelle legislature degli anni Duemila almeno fino al 2013, il procedimento che porta dallo svolgimento delle elezioni alla formazione del governo ${ }^{58}$ - che ha come protagonisti il presidente della repubblica e, dall'altro lato, i partiti - si trasforma progressivamente e diviene più snello e (per conseguenza) più breve che in passato. Ciò dipende dal fatto che, dopo il cambiamento del sistema elettorale in senso maggioritario ed i connessi mutamenti del quadro politico che si sono descritti nel paragrafo precedente, da una parte, le elezioni esprimono un risultato (cioè dei rapporti di forza parlamentari) chiaro e già sufficientemente delineato; dall'altra parte, i partiti stringono le alleanze - ed indicano, in particolare, il loro candidato alla presidenza del consiglio - fin da prima del momento elettorale, e si comportano poi di conseguenza. A partire dalle elezioni politiche del 2013, ed in particolare a seguito delle elezioni della primavera del 2018, si ha invece, (anche) da questo punto di vista, un'inversione di tendenza: tanto la formazione del governo Letta dopo le prime, quanto più la genesi del governo Conte (I) dopo le seconde ${ }^{59}$, risultano infatti processi lunghi e "faticosi", il che appare spiegabile con la circostanza che tali consultazioni elettorali - a differenza delle precedenti - non esprimono un risultato ben definito, in ragione del cambiamento 
intervenuto nel quadro politico e del passaggio da un sistema "bipolare" ad un assetto caratterizzato dalla presenza di tre coalizioni/soggetti politici dal peso elettorale simile.

In secondo luogo, emerge l'indebolimento - notevole - del rapporto di fiducia durante il corso delle legislature: tendenza in contraddizione, apparentemente, con quella sopra evidenziata (almeno per quanto riguarda il periodo 1993/2013), o comunque tale da attenuarne l'impatto sistematico. Tale indebolimento del rapporto di fiducia - che in molti casi porta a delle vere e proprie crisi di governo (v. oltre, § 2.3) - si manifesta in concreto in due principali fenomeni. Da una parte (dal lato dei governi), nell'abuso della posizione della questione di fiducia su specifici provvedimenti, allo scopo di "costringere" il parlamento ad approvarli60: le statistiche parlamentari ${ }^{61}$ rivelano che nelle legislature dopo il 1993 e, soprattutto, negli anni a noi più vicini - il ricorso dei governi (di ogni tendenza politica) alla questione di fiducia ha assunto dimensioni sempre più ragguardevoli e, come tali, preoccupanti ${ }^{62}$. Dall'altra parte (dal lato, questa volta, delle camere, ed in particolare dei partiti di maggioranza), vi è la messa in discussione frequente del rapporto fiduciario con l'esecutivo, attraverso voti contrari alle proposte di questo ${ }^{63}$ e la sollecitazione ricorrente di cc.dd. «verifiche»: una vera e propria costante, allo stesso modo, delle legislature dal 1994 ad oggi. Tutto ciò è indice di una grande (e non "fisiologica") difficoltà del circuito partiti- coalizioni politiche a mantenere una relazione con l'esecutivo connotata da stabilità, rispetto all'inizio della legislatura. Questo, peraltro, dipende a sua volta dall'elevata conflittualità interna al sistema dei partiti in tutto il periodo esaminato (che oggi pare addirittura in fase di accentuazione), della quale si è detto nel paragrafo precedente.

In terzo (ed ultimo) luogo, con riferimento all'istituto della mozione di sfiducia (previsto dall'art. 94 ultimo comma della Costituzione italiana), negli anni più vicini alla "svolta" del 1993 si assiste ad un utilizzo abbastanza frequente - da parte, evidentemente, delle opposizioni parlamentari della mozione di sfiducia nei confronti dell'intero governo: l'unica forma, peraltro, che allora si riteneva consentita dalla Costituzione ${ }^{64}$. Le mozioni di sfiducia vengono comunque, di norma, respinte: le crisi di governo extra-parlamentari sono del tutto prevalenti nell'esperienza istituzionale 
italiana (v. oltre, § 2.3). Viceversa, a partire dal «caso Mancuso» del $1995^{65}$ - e dalla relativa sentenza n. 7/1996 della corte costituzionale, che ha giudicato ammissibile anche questo tipo di sfiducia ${ }^{66}$-, vengono presentate più frequentemente mozioni di sfiducia individuali: nei confronti, cioè, di singoli ministri ${ }^{67}$. Questa tendenza si accentua negli anni a noi più vicini, in contemporanea al consolidamento della dinamica maggioritaria e "bipolare" del sistema politico (di cui si è detto sopra): la mozione di sfiducia individuale, in tale contesto, viene utilizzata dall'opposizione per mettere in difficoltà il governo nel suo insieme, e la coalizione politica che lo sostiene, dato che essi sono posti di fronte all'alternativa tra proteggere il ministro "sfiduciando" - talvolta in situazioni anche delicate ed imbarazzanti - oppure "scaricarlo" (com'era avvenuto proprio nel «caso Mancuso»), costringendolo a dimettersi. Entrambe le soluzioni possono provocare contraccolpi sulla maggioranza politica, soprattutto se sono coinvolti ministri di rilievo. Si deve comunque ricordare che, fino ad oggi, nessuna mozione di sfiducia individuale è stata in concreto approvata dalle camere.

\section{LE CRISI DI GOVERNO E LO SCIOGLIMENTO ANTICIPATO DELLE CAMERE}

Il terzo "elemento materiale" da analizzare ai fini della ricostruzione dell'evoluzione della forma di governo in Italia dopo il 1993 è costituito dalle crisi di governo. Questo elemento, appunto in chiave sistematicoricostruttiva, deve essere peraltro considerato in relazione all'esercizio ${ }^{68}$ del potere di scioglimento anticipato delle camere, attribuito al presidente della Repubblica dall'art. 88 della Costituzione italiana.

In proposito, nel periodo oggetto di analisi sono da segnalare diverse evidenze fattuali, che concorrono nel loro insieme a caratterizzare la forma di governo parlamentare italiana ${ }^{69}$ :

-a) la prevalenza numerica delle crisi «extraparlamentari» - cioè quelle in cui il governo si dimette non a seguito di un voto di sfiducia del parlamento, ai sensi dell'art. 94 Cost. - su quelle «parlamentari» (in continuità con la fase precedente di storia repubblicana): le uniche crisi 
«parlamentari» del periodo considerato sono due, nel 1998 e nel 2008 (in entrambi i casi Presidente del consiglio Romano Prodi) ${ }^{70}$;

-b) la diminuzione peraltro - in termini assoluti - del numero di crisi di governo rispetto al periodo precedente (dal 1948 al 1993): ciò, nonostante le difficoltà del sistema politico esposte nei paragrafi precedenti, è stato ritenuto dalla dottrina indice di un «affinamento sempre maggiore dei meccanismi bipolari [e di una] sempre maggiore stabilità delle coalizioni di Governo» ${ }^{71}$. Si deve tuttavia ricordare che le due legislature a noi più vicine (2013/2018 e dal 2018, attualmente in corso) esprimono, anche da questo punto di vista, un'inversione di tendenza: nella prima vi sono state due crisi di governo (Letta e Renzi), con sostanziale continuità della maggioranza politica successiva; nella seconda vi è stata già una crisi in poco più di un anno (Conte I), con conseguente cambiamento della maggioranza (da MoVimento 5 Stelle - Lega a MoVimento 5 Stelle - Partito democratico ed altri partiti di centrosinistra);

-c) la tendenza alla c.d. «parlamentarizzazione delle crisi» ${ }^{72}$ - cioè al "trasferimento" nelle aule parlamentari, mediante lo svolgimento di un apposito dibattito, delle crisi politiche maturate senza proposizione di mozioni di sfiducia o votazioni contrarie al governo in carica -, favorita soprattutto dai presidenti della repubblica; e, più in generale, la tendenza alla «parlamentarizzazione» delle procedure di verifica della sussistenza del rapporto fiduciario, anche attraverso la prassi della c.d. «questione di fiducia virtuale» ${ }^{73}$;

-d) il mutamento di condotta dei partiti politici durante il corso delle legislature, rispetto al loro posizionamento elettorale ed alla scelta di sostenere o meno l'esecutivo formato dopo le elezioni: da questa tendenza consegue spesso il cambiamento della maggioranza politica e, "a cascata", la crisi e sostituzione del governo stesso (v. già sopra, $\S \S 2.1-2.2$ ), come confermato - da ultimo - dalla crisi del governo Conte I e dal passaggio al governo Conte II (con stesso premier, ma diversa maggioranza politica ${ }^{74}$ ) avvenuti nell'attuale legislatura, nell'estate del 2019;

-e) la semplificazione e la velocizzazione, peraltro, nel contesto sopra richiamato, delle procedure e dei passaggi istituzionali correlati alla crisi di governo: salvo rare eccezioni ${ }^{75}$, non si ripete la prassi, frequente nella «Prima Repubblica», delle crisi di governo cc.dd. «al buio»; 
-f) l'oscillazione in ordine all'esercizio - e, correlativamente, alla concezione teorica - del potere di scioglimento anticipato delle camere da parte del presidente della repubblica: la prassi istituzionale di questo ventennio attesta una sostanziale "fluidità" di tale potere, che in parte viene esercitato (o anche solo prospettato dai presidenti) come «extrema ratio» di fronte all'accertata impossibilità di formare una qualsiasi maggioranza politica dopo le dimissioni del governo; in parte viene "reclamato" dagli attori politici - e talvolta anche esercitato in concreto (v. poco oltre) - come "conseguenza automatica" della crisi dell'esecutivo e della maggioranza parlamentare scaturiti dalle elezioni, in un contesto che (almeno fino al 2013/2018), tende a divenire maggioritario e "bipolare";

-g) infine - in stretta connessione al fenomeno da ultimo richiamato -, l'emersione in primo piano del ruolo del presidente della repubblica nella gestione delle crisi di governo, rispetto alla già pur rilevante posizione da esso detenuta nel periodo antecedente al 1993: ciò avviene, in particolare, con le due presidenze Napolitano, dal 2006 al 2013 e dal 2013 al $2015^{76}$.

Le tendenze sopra ricordate si ricavano dall'analisi del concreto svolgimento delle crisi di governo nel periodo dopo il $1993^{77}$ e, dall'altro lato, degli scioglimenti anticipati delle camere che in esso sono (o, come subito si preciserà, non sono) stati disposti. A questo riguardo, non potendosi qui, per ragioni di spazio, ripercorrere nel dettaglio tutte le vicende rilevanti, ci si limita a ricordare che dal 1993 al 2018 (inizio dell'attuale legislatura) gli scioglimenti anticipati sono stati tre ${ }^{78}$ - su sette legislature complessive - e sono riconducibili a due distinte categorie dogmatiche: - quello del gennaio 1994 (presidente della repubblica Scalfaro), alla categoria degli scioglimenti «sostanzialmente presidenziali» ${ }^{79}$ (anche se, per la verità, non mancano in dottrina ricostruzioni diverse ${ }^{80}$ ); - quelli del febbraio 1996 e del febbraio 2008 (rispettivamente, presidenti Scalfaro e Napolitano), invece, alla categoria - più frequente - dello scioglimento come «extrema ratio» (o «di funzionalità»), imputabile al presidente della repubblica solo dal punto di vista formale, ma nella sostanza alla volontà delle forze politiche presenti in parlamento di porre termine alla legislatura ${ }^{81}$.

Oltre agli scioglimenti anticipati veri e propri, risultano peraltro significativi ai fini della presente indagine i casi di mancato scioglimento delle camere da parte dei presidenti della Repubblica; e ciò nonostante 
l'esplicita richiesta in tal senso loro formulata dalle forze politiche, o almeno da alcune di esse (solitamente quelle di opposizione, e/o già di maggioranza). Nel periodo qui esaminato questo è avvenuto diverse volte: nel 1994, dopo la crisi del governo Berlusconi I (presidente della repubblica Scalfaro); nel 2000, dopo le dimissioni del secondo governo D’Alema (presidente Ciampi); nel febbraio 2007 e nel gennaio 2008, dopo le crisi del governo Prodi II (presidente Napolitano); nel novembre 2011, dopo le dimissioni del governo Berlusconi IV (presidente sempre Napolitano) ${ }^{82}$; nel dicembre 2016, dopo le dimissioni del governo Renzi a seguito del referendum costituzionale svoltosi il 4 dicembre (presidente Mattarella); da ultimo, in questa legislatura, nell'agosto del 2019, dopo le dimissioni del governo Conte I in seguito alla mozione di sfiducia presentata dalla Lega di Salvini ${ }^{83}$, che ha invocato con forza - insieme ai partiti di centrodestra - l'indizione di nuove elezioni (presidente sempre Mattarella). Tali circostanze manifestano, come si è rilevato sopra, una divergenza in ordine alla concezione del potere di scioglimento: se potere esercitabile solo nel caso di impossibilità di formare una qualsiasi maggioranza politica (anche diversa da quella formatasi dopo le elezioni: scioglimento come «extrema ratio»), oppure da esercitarsi - alla luce dell'introdotto sistema elettorale maggioritario ${ }^{84}$ - ogniqualvolta la maggioranza politica formatasi dopo le elezioni venga meno (prospettandosi come necessario, in tal caso, il «ritorno agli elettori» ${ }^{85}$ ). La prassi istituzionale al riguardo inclina spesso, peraltro, verso il mezzo. I presidenti della Repubblica, infatti, dopo la crisi di governo si dichiarano prevalentemente "liberi" di ricercare in parlamento una maggioranza politica diversa, in modo da evitare lo scioglimento anticipato. Tuttavia, nel fare ciò, essi sembrano più "vincolati" rispetto al passato dal significato maggioritario che viene attribuito al voto: questo sconsiglia - in concreto - la formazione di maggioranze politiche radicalmente differenti da quelle scaturite dalle elezioni, ed i presidenti tendono ad agire di conseguenza ${ }^{86}$ (non sempre però: si pensi, per esempio, alla soluzione delle crisi di governo del 1994, del 2011 e - da ultimo - del 2019, tutte caratterizzate da un cambiamento sensibile di maggioranza parlamentare). 


\section{IL RUOLO POLITICO- COSTITUZIONALE DEL GOVERNO ED IL SUO RAPPORTO, IN PARTICOLARE, CON GLI ORGANI DI GARANZIA}

Per quanto riguarda, infine, il ruolo esercitato in concreto dal governo e - in particolare - il rapporto tra tale organo e quelli di garanzia costituzionale, nel periodo dal 1993 ad oggi è possibile rilevare, in primo luogo, la tendenza al complessivo rafforzamento dell'esecutivo nel sistema politico e costituzionale ${ }^{87}$. Rispetto alla lunga fase precedente di storia repubblicana italiana (la c.d. «Prima repubblica» alla quale si è fatto più volte cenno), il governo acquista un'influenza sempre maggiore nel sistema - in conseguenza dell'affermazione del principio maggioritario e della progressiva stabilizzazione delle dinamiche istituzionali ad esso correlate ${ }^{88}$ -, fino a diventare, negli anni Duemila, il vero e proprio «baricentro» del sistema politico: cioè l'organo al cui interno vengono elaborate ed attuate le decisioni politiche fondamentali (quello che la dottrina definisce l'«indirizzo politico di maggioranza» ${ }^{89}$ ), a discapito del parlamento, il quale tende invece (secondo un'altra fortunata espressione dottrinale) a «declinare ${ }^{90}$. Questa tendenza (in Italia come, del resto, negli altri Paesi) è stata ulteriormente rafforzata, da ultimo, dall'“emergenza Covid", che ha «concentrato» in capo all'Esecutivo - in parte fisiologicamente - le decisioni relative alla gestione dell'emergenza stessa ed ai gravissimi problemi (economici in primo luogo) ad essa correlati.

Questo "spostamento di potere politico" (ravvisabile, peraltro, in tutte le odierne democrazie parlamentari) è dimostrato, principalmente, dall'analisi delle dinamiche del procedimento legislativo e, nello specifico, dai dati riguardanti l'attività di produzione delle fonti primarie (che attuano, appunto, l'«indirizzo politico di maggioranza»). Al riguardo, nel periodo temporale qui considerato emergono le seguenti evidenze ${ }^{91}$ : -a) l'incremento quantitativo degli atti normativi primari adottati dal governo (decreti legislativi e decreti- legge, ai sensi, rispettivamente, degli artt. 76 e 77 Cost.) rispetto alle leggi formali approvate dal parlamento; -b) la prevalenza - sempre sul piano quantitativo - dell'iniziativa legislativa del governo all'interno delle camere rispetto a quella degli altri organi previsti dalla Costituzione (singoli parlamentari in primis) ${ }^{92}$; -c) l'espansione 
soprattutto dell'utilizzo del decreto- legge, tramite il quale vengono adottate nel corso degli anni delle vere e proprie normative «di sistema» (come le manovre economiche "ex Finanziaria" degli anni dal 2008 in poi ${ }^{93}$ ); -d) sul piano, questa volta, "qualitativo", l'approvazione con atti riconducibili al governo (decreti- legge, decreti legislativi, leggi formali di iniziativa governativa) dei più importanti e qualificanti provvedimenti di riforma $^{94}$; -e) l'estrema dilatazione del tempo dedicato dalle camere alla discussione dei provvedimenti presentati dall'esecutivo, rispetto a quelli di iniziativa parlamentare; $-f$ ) l'intervento fortemente condizionante (quando non decisivo) dello stesso esecutivo nella discussione parlamentare dei provvedimenti legislativi, tramite la presentazione di cc.dd. «maxiemendamenti» - che sostituiscono l'intero testo in corso di discussione nelle camere - e (molto spesso, contemporaneamente ${ }^{95}$ ) la posizione della questione di fiducia - che costringe, di fatto, il parlamento ad approvare tale testo senza ulteriore dibattito -.

Questi ultimi strumenti “tecnici”, volti ad incidere pesantemente sul procedimento legislativo, sono stati imputati dagli esecutivi (di ogni colore politico) - non del tutto infondatamente - alle carenze dei regolamenti parlamentari in Italia, che non garantirebbero al governo "tempi certi" per far approvare le sue proposte ${ }^{96}$. Il loro vasto utilizzo nel periodo dopo il 1993, tuttavia, è indice di un'altra tendenza generale, in qualche modo opposta a quella innanzi segnalata: la difficoltà dell'esecutivo a far approvare i suoi provvedimenti in parlamento tramite le procedure ordinarie. Il governo, in sostanza, diviene sì l'organo più rilevante sul piano politico; ma lo fa molto spesso derogando alle procedure ordinarie di adozione degli atti normativi previste dalla Costituzione e dai regolamenti delle camere ${ }^{97}$. Ciò - soprattutto nel primo decennio degli anni Duemila, quello di maggiore affermazione del parlamentarismo maggioritario - ha innescato dei notevoli conflitti tra l'esecutivo e gli organi di garanzia (presidente della repubblica, corte costituzionale, giudici comuni), incaricati di assicurare il rispetto delle citate norme e procedure costituzionali ${ }^{98}$.

Come esempi pratici di tali situazioni di conflitto si possono richiamare (anche qui, in sintesi): -a) gli interventi dei presidenti della repubblica (soprattutto nelle presidenze Ciampi e Napolitano): rinvii delle leggi ${ }^{99}$ (e in un caso, di un decreto- legge ${ }^{100}$ ), ma anche ripetuti 
richiami al governo e messaggi alle camere ed ai loro presidenti ${ }^{101}$; -b) alcune importanti pronunce della corte costituzionale, che hanno dichiarato costituzionalmente illegittimi dei decreti- legge - o meglio, delle leggi di conversione di decreti- legge - per mancanza dei presupposti previsti dall'art. 77 della Costituzione («straordinaria» necessità ed urgenza ed omogeneità del contenuto del d.l.: sentenze n. 360/1996, in tema di «reiterazione» del d.l.; n. 171/2007; n. 28/2008; n. 22/2012; n. $220 / 2013^{102}$ ); -c) per quanto riguarda i giudici comuni, la rimessione alla corte costituzionale delle citate questioni di costituzionalità dei decretilegge e - più in generale - la censura di numerosi provvedimenti dei governi per violazione di altri parametri costituzionali (nel quadro del conflitto tra il potere politico e la magistratura, che ha rappresentato invero un'altra "cifra" caratterizzante l'intero periodo di storia repubblicana qui oggetto di analisi).

In definitiva: la crescita dell'influenza dell'esecutivo nel sistema politico e costituzionale italiano - che induce a ricondurre la forma di governo al parlamentarismo «maggioritario» ${ }^{103}$ - è avvenuta, nel periodo successivo allo snodo del 1993, in modo non conforme alle disposizioni costituzionali, e spesso anzi "a prezzo" della loro distorsione interpretativa (o addirittura, violazione esplicita). Questo ha generato le diffuse tensioni con gli organi di garanzia, innanzi ricordate.

\section{LE TENDENZE GENERALI DELLA FORMA DI GOVERNO IN ITALIA DOPO IL 1993; L'EMERSIONE IN PRIMO PIANO DEL PRESIDENTE DELLA REPUBBLICA}

L'analisi dei diversi "elementi materiali" della forma di governo in Italia - nella loro evoluzione dopo il 1993 - svolta nei precedenti paragrafi consente, a questo punto, di ricavare alcune tendenze generali. Muovendo da esse, si procederà (nel prossimo paragrafo) ad una valutazione complessiva dell'evoluzione della forma di governo stessa, con riguardo anche al suo inquadramento sistematico in una delle «varianti» tipiche del parlamentarismo ricordate nella parte iniziale di questo scritto. 
Le tendenze generali ("materiali"104) che emergono sono essenzialmente cinque:

-1) l'aggregazione dei partiti in coalizioni prima dello svolgimento delle elezioni; e, correlativamente, l'indicazione preliminare del candidato presidente del consiglio da parte delle coalizioni stesse;

-2) la "rottura"/disgregazione dei partiti e - più sovente - delle coalizioni dopo lo svolgimento delle elezioni, a cui conseguono la crisi del rapporto di fiducia maggioranza- governo e le dimissioni di quest'ultimo; spesso durante la legislatura si verifica anche il cambiamento della maggioranza politica, oltre che del governo;

-3) la difficoltà dei rapporti tra il parlamento ed il governo, sia per quanto riguarda il rapporto di fiducia (come si è appena visto) che per ciò che concerne l'esercizio concreto delle funzioni dei due organi;

-4) la "tensione" - e talvolta, il conflitto istituzionale vero e proprio - tra il governo (e più in generale gli organi «di indirizzo») e gli organi di garanzia costituzionale;

-5) l'emersione in primo piano del ruolo - e della figura costituzionale - del presidente della Repubblica.

Su quest'ultima tendenza/mutamento complessivo, per la sua importanza sistematica, è necessario fermare un momento l'attenzione, pur non potendosi esaminare nel dettaglio tutte le vicende politicocostituzionali che ne giustificano la sottolineatura, per ragioni di spazio.

Essa - per il vero - affonda le radici nell'ultima fase del periodo di storia repubblicana antecedente al 1993, nel quale le presidenze Pertini e Cossiga ${ }^{105}$ si erano già allontanate dal modello «notarile» che, in generale, si riteneva essersi affermato nei primi anni della Repubblica. E del resto, uno dei caratteri tipici dell'organo presidenziale nella forma di governo italiana, secondo la dottrina costituzionalistica, è l'“elasticità" dei suoi poteri (definiti, con immagine efficace, «a fisarmonica»), cioè l'attitudine ad espandersi o a restringersi a seconda del contesto politico e, in particolare, del (rispettivamente) cattivo o buon funzionamento del circuito d'indirizzo parlamento- governo ${ }^{106}$.

Tuttavia, nel periodo qui specificamente considerato pare determinarsi una soluzione di continuità rispetto al passato: sotto il profilo “quantitativo", poiché l'esercizio da parte del presidente di poteri 
particolarmente ampi (rispetto alla "fisiologia" di una forma di governo parlamentare) non è più un evento "straordinario" e transitorio, ma si protrae con continuità fino quasi a diventare un dato strutturale del sistema; sotto il profilo "qualitativo", dall'altro lato, poiché i poteri e la figura costituzionale del presidente della repubblica tendono anche a cambiare natura, evolvendosi (sia pure in modo non costante) verso una maggiore partecipazione alla definizione ed all'attuazione dell'indirizzo politico. Questa constatazione ha portato molti commentatori - e, per quanto qui interessa, alcuni costituzionalisti ${ }^{107}$ - ad affermare che la forma di governo italiana avrebbe subito, nel periodo in esame, addirittura un «mutamento tacito», in senso appunto presidenziale ${ }^{108}$.

Scendendo più nello specifico, dal 1993 ad oggi vi sono - anche in questo caso - diverse evidenze che consentono di ritenere in atto, se non proprio un mutamento del tipo di forma di governo, quantomeno un'evoluzione del parlamentarismo caratterizzata dalla notevole crescita di importanza (e dei poteri) del presidente della Repubblica. Esse prevalentemente sono state ricordate nei precedenti paragrafi:

-a) la centralità assunta dal presidente nella formazione dei governi e (soprattutto) durante le crisi di governo: il presidente della Repubblica, salvo rari casi, non si limita a "sovraintendere" ed a "coordinare" le procedure volte alla formazione del governo ed alla verifica della possibilità di instaurare il rapporto fiduciario, ma esercita un ruolo "attivo" e condizionante (le scelte dei partiti politici) su entrambi i fronti, con strumenti diversi;

-b) il crescente "interventismo" del presidente della Repubblica nello svolgimento quotidiano dell'attività politica e, più in generale, nella vita pubblica: esso si realizza - prima ancora che attraverso l'esercizio dei suoi poteri «formali» previsti dalla Costituzione - sempre più tramite atti «informali», come le cc.dd. «esternazioni»; i richiami alle forze politiche ad adottare (o a valutare la possibilità di adottare) determinati provvedimenti e misure; i «comunicati stampa» e le «note» pubblicati sul sito Internet della Presidenza della Repubblica, e perciò direttamente accessibili a tutti;

-c) in particolare, l'intervento rilevante del presidente - tramite i suoi atti «formali» - sui provvedimenti adottati dagli organi politico- 
rappresentativi, che spesso si espongono a censure di incostituzionalità (rinvii delle leggi e messaggi alle camere);

-d) infine (ma non ultimo ${ }^{109}$ ), l'“impulso" - sempre da parte del presidente - ai processi di riforma istituzionale e, in particolare, costituzionale: tutti i presidenti che si sono succeduti dal $1993^{110}$, invero, hanno espresso (seppure con accenti diversi) una posizione favorevole all'adozione di riforme - anche costituzionali - volte a ridefinire l'assetto istituzionale italiano; e ciò sul presupposto che l'assetto tradizionale, dopo i mutamenti politici intervenuti appunto dal 1993, non fosse più adeguato a perseguire efficacemente i propri fini. Il presidente Napolitano, nell'ultima parte del suo primo mandato e nel momento della sua (storica) rielezione del 2013, si è spinto ancora più avanti: in un contesto di gravissima difficoltà del sistema politico e di paralisi decisionale dei partiti della «Seconda repubblica», coniugata ad una situazione di perdurante crisi economica e di legittimazione dell'Italia nel consesso unitario europeo, ha nominato una commissione di esperti, incaricandola di elaborare proposte di «misure dirette ad affrontare ... la crisi del sistema istituzionale» (i cc.dd. «saggi», che hanno depositato la loro Relazione finale il 12 aprile 2013)111; poi - nel discorso tenuto davanti alle camere in seduta comune subito dopo la sua rielezione - ha addirittura legato esplicitamente la rielezione e la sua permanenza nella carica alla realizzazione delle riforme istituzionali e costituzionali, definite «improrogabili» 112 .

Questo orientamento - al di là delle possibili valutazioni di merito sembra dimostrare in modo particolare la "mutazione qualitativa" occorsa ai poteri ed al ruolo dell'organo presidenziale nel sistema costituzionale italiano nel periodo dal 1993 ad oggi: da «notarili» e di pura garanzia costituzionale a (almeno in parte) di «indirizzo politico» ${ }^{113}$. Si tratta di un dato certamente rilevante per la ricostruzione complessiva della forma di governo ${ }^{114}$.

Si deve ricordare, infine, che durante questo periodo anche la giurisprudenza costituzionale - in particolare con la sentenza n. 1/2013, riguardante il conflitto di attribuzioni sorto tra il presidente Napolitano e la procura della Repubblica di Palermo in ordine all'utilizzo di alcune intercettazioni telefoniche del presidente ${ }^{115}$ - ha sancito la centralità ("attiva") del presidente della Repubblica nel sistema costituzionale. La 
corte costituzionale ha definito il presidente, in modo significativo dal punto di vista di questo lavoro, organo "garante dell'equilibrio costituzionale» e della «coesione e dell'armonico funzionamento dei poteri, politici e di garanzia, che compongono l'assetto costituzionale della Repubblica ... organo di moderazione e di stimolo nei confronti di altri poteri, in ipotesi tendenti ad esorbitanze o ad inerzia ... Tutti i poteri del Presidente della repubblica - ha poi affermato la corte nella sentenza citata - hanno ... lo scopo di consentire allo stesso di indirizzare gli appropriati impulsi ai titolari degli organi che devono assumere decisioni di merito, senza mai sostituirsi a questi, ma avviando e assecondando il loro funzionamento, oppure, in ipotesi di stasi o di blocco, adottando provvedimenti intesi a riavviare il normale ciclo di svolgimento delle funzioni costituzionali» ${ }^{116}$.

\section{UNA VALUTAZIONE DI SINTESI: LA FORMA DI GOVERNO ITALIANA DAL 1993 AD OGGI ED IL SUO INQUADRAMENTO NELLE “SOTTOCATEGORIE” TIPICHE DELLA FORMA DI GOVERNO PARLAMENTARE}

A partire dalle tendenze generali sopra evidenziate, si può allora procedere ad una valutazione di sintesi circa la natura e l'evoluzione della forma di governo in Italia dal 1993 ai giorni nostri, cercando inoltre (come preannunciato) di classificarla in una delle "sottocategorie" - o «varianti» - tipiche del parlamentarismo.

A tal fine, è necessario qui ricordare che prima della "svolta" politicoistituzionale del 1993 la forma di governo italiana veniva definita dalla dottrina parlamentarismo «a multipartitismo esasperato» (Elia ${ }^{117}$ ), o «compromissorio», o «a prevalenza del Legislativo» (Di Giovine ${ }^{118}$ ). Si intendeva sottolineare - con queste qualificazioni - la centralità dei partiti e delle loro dinamiche (sia interne che "esterne": loro rapporti reciproci) in tale forma di governo; dall'altro lato (e quale conseguenza diretta), la «prevalenza» (o il «primato») del parlamento sul governo e la debolezza ed instabilità degli esecutivi. Ciò in un contesto in cui, per una precisa scelta del Costituente, la forma di governo parlamentare era (come è tuttora) disciplinata con poche disposizioni costituzionali, dal 
contenuto scarno ${ }^{119}$ : era quella dinamica dei rapporti politici a determinare in concreto la forma di governo.

Rispetto a questo "punto di partenza", il periodo successivo al referendum elettorale del 1993 e a noi più vicino registra, come si è visto nel paragrafo precedente, diversi fenomeni e tendenze "di rottura", ma anche altri che esprimono una sostanziale continuità del parlamentarismo italiano.

I primi fenomeni sono rappresentati (1) dall'aggregazione dei partiti (peraltro quasi del tutto diversi da quelli del periodo precedente, e questo è un dato estremamente rilevante ${ }^{120}$ ) in coalizioni politiche prima delle elezioni, con la contestuale indicazione del nome del candidato Premier di ogni coalizione ${ }^{121}$; (2) dal sensibile rafforzamento del governo nel sistema politico- costituzionale e, al suo interno, del presidente del consiglio ${ }^{122}$; (3) da una "tensione" tra il governo (e più in generale gli organi «di indirizzo») e gli organi di garanzia più elevata rispetto al passato (della quale si sono precedentemente indicati alcuni aspetti significativi) e che, rispetto ad altre forme di governo parlamentari, esibisce - come si è visto - una componente "patologica"; (4) dall'emersione, infine, del ruolo e della figura costituzionale del presidente della repubblica (che si ritrova anche in alcune delle proposte di revisione costituzionale della forma di governo stessa - da parlamentare a presidenziale, appunto, o almeno «semipresidenziale» - presentate nel corso del periodo qui esaminato).

Queste tendenze, complessivamente considerate, porterebbero a ritenere che la forma di governo italiana si sia evoluta verso un parlamentarismo «maggioritario», a caratterizzazione sostanzialmente «bipolare» se non bipartitica (o - volendo richiamare ancora la classificazione di Elia, e tenendo conto della persistenza nel sistema politico di partiti diversi - «a multipartitismo temperato» ${ }^{123}$ ) e $a$ prevalenza dell'esecutivo. Vi sono tuttavia anche, nel periodo dal 1993 ad oggi, dei fenomeni e tendenze di segno diverso, ispirati - come si è detto - a continuità rispetto alle caratteristiche del precedente regime parlamentare. Si tratta in particolare: (1) dell'estrema conflittualità interna ai partiti, che si trasferisce anche alle coalizioni quale effetto delle notevoli differenze esistenti tra i partiti stessi (indotti a coalizzarsi dal sistema elettorale maggioritario in vigore almeno fino al 2017) ${ }^{124}$; (2) 
della (correlata) difficoltà dei rapporti tra il parlamento ed il governo, di cui si sono indicati ${ }^{125}$ gli aspetti più rilevanti (e che in genere non si riscontra nei parlamentarismi «maggioritari»).

In definitiva, l'evoluzione verso il parlamentarismo «maggioritario» (o «a multipartitismo temperato», o a prevalenza dell'esecutivo) è avvenuta in Italia, nel periodo successivo al 1993, in modo parziale; "coatto", perché essa è stata determinata dal cambiamento delle regole elettorali imposto dal referendum popolare del 18 aprile 1993, più che dalla spontanea volontà degli attori politici ${ }^{126}$; $\mathrm{e}$ - infine - contraddittorio, permanendo nel sistema dei significativi elementi di "conservazione" (o se si preferisce, di "trascinamento" e "resistenza") del regime precedente. E negli anni a noi più vicini - in conseguenza degli ulteriori cambiamenti del sistema politico dovuti all'affermazione del MoVimento 5 Stelle, e della reintroduzione di un sistema elettorale prevalentemente proporzionale ad opera della legge n. 165/2017 - la forma di governo sembra essere ritornata ad una connotazione in qualche modo assimilabile a quella del primo periodo di storia repubblicana, sopra ricordata. Risulta perciò difficile classificare in termini univoci l'attuale forma di governo italiana (tanto più in seguito alle vicende più recenti): essa, più che rientrare in una delle precise "sottocategorie" (o «varianti») del parlamentarismo individuate dalla dottrina, appare una forma di governo parlamentare "ibrida", che - allo stato - esibisce elementi tanto dell'una (parlamentarismo «maggioritario») quanto dell'altra (parlamentarismo «compromissorio») «variante» ${ }^{127}$.

Del resto, si è osservato all'inizio di questo lavoro che le classificazioni astratte delle forme di governo - seppure utili in chiave ricostruttiva - spesso non risultano del tutto adeguate a descrivere l'effettività dei regimi politici. 


\section{L'INTERPRETAZIONE DELLE DISPOSIZIONI COSTITUZIONALI SULLA FORMA DI GOVERNO ED IL RAPPORTO TRA QUESTE REGOLE FORMALI E LA PRASSI DEGLI ORGANI COSTITUZIONALI}

Passando ora ad esaminare più specificamente il modo in cui le disposizioni costituzionali sulla forma di governo in Italia sono state interpretate dagli attori politici ed istituzionali dopo il 1993 - e dunque il rapporto intercorrente tra queste regole formali e la prassi degli organi costituzionali, conformemente alla scansione del lavoro presentata nel primo paragrafo -, occorre rilevare, in primo luogo, che il quadro costituzionale è caratterizzato da poche regole, dal contenuto minimo. Gli artt. 92 e seguenti della Carta, infatti, dispongono solo che il presidente del consiglio ed i ministri siano nominati dal presidente della repubblica e che il governo entro dieci giorni dalla nomina debba presentarsi alle camere per ottenere la fiducia ${ }^{128}$; gli artt. 94 e 88 prevedono, rispettivamente, l'istituto della mozione di sfiducia e dello scioglimento anticipato delle camere da parte del presidente della repubblica, senza tuttavia indicarne i presupposti (in particolare per lo scioglimento anticipato) e le conseguenze (in particolare per la mozione di sfiducia, al di là della previsione - peraltro implicita ${ }^{129}$ - dell'obbligo di dimissioni del governo sfiduciato). Tale stringatezza delle regole costituzionali, come è noto, non è casuale: essa risponde ad una scelta ben precisa dell'Assemblea costituente ${ }^{130}$, secondo cui la forma di governo parlamentare non doveva essere disciplinata in modo analitico, così da permettere alla prassi successiva (cioè alle relazioni future tra i partiti) di determinarne contenuto ed evoluzione.

Va inoltre osservato che nel periodo dopo il 1993 (come già nella prima fase di storia repubblicana italiana) le citate disposizioni costituzionali non hanno subito modifiche, nonostante le ripetute proposte di riforma che sono state presentate ${ }^{131}$. La principale riforma che ha inciso sulla forma di governo, come si è visto - l'introduzione del sistema elettorale maggioritario ${ }^{132}$-, è stata approvata con legge ordinaria (a seguito del referendum popolare svoltosi il 18 aprile 1993) ${ }^{133}$.

Ciò precisato, nel periodo in esame è possibile ravvisare una sostanziale continuità anche nell'interpretazione ed applicazione delle 
disposizioni costituzionali di cui trattasi. Non si registrano nella prassi dei rilevanti mutamenti interpretativi, ma solo degli "adattamenti", per così dire, al mutato contesto politico maggioritario scaturito dalla riforma elettorale del 1993 e persistente - come si è visto - fino almeno alla legislatura aperta dalle elezioni politiche del 2013. In altri termini, gli "interpreti istituzionali" della Costituzione in tema di forma di governo - in primo luogo il presidente della Repubblica, "garante supremo" del sistema ${ }^{134}$ - hanno continuato a dare una lettura del testo costituzionale aderente ad un parlamentarismo «compromissorio» e «a prevalenza del legislativo», come quella che si era affermata nei decenni precedenti di storia della Repubblica.

Non è possibile qui soffermarsi sulle singole disposizioni e sulle loro rispettive vicende interpretative ${ }^{135}$. Solo a titolo di esempio, si può ricordare che l'art. 88 Cost. - sul potere di scioglimento anticipato delle camere - tende ancora ad essere interpretato dai presidenti della Repubblica (e dalla prevalente dottrina) nel senso di prevedere un potere da esercitarsi (solo) nell'ipotesi di accertata impossibilità di formare un governo, e quindi una qualunque maggioranza politica (scioglimento come «extrema ratio»), anziché nei casi di estinzione della maggioranza politica/ coalizione risultata vincitrice alle elezioni, pur in un contesto maggioritario e (che tende a divenire) «bipolare». Gli scioglimenti anticipati disposti nel dopo il 1993 (1996 e 2008 ${ }^{136}$ ) sono espressione di questa lettura.

Dunque pare possibile affermare: $-a$ ) che il mutamento del sistema politico in senso maggioritario, avvenuto a partire dal citato referendum elettorale e fino almeno al 2013, è stato per lo più "svalutato" in sede di applicazione concreta delle disposizioni costituzionali sulla forma di governo; - $b$ ) e che quindi l'evoluzione della forma di governo - che pure, come si è visto poco sopra, vi è stata nel periodo dopo il 1993 - è stata un'evoluzione (b.1) a Costituzione invariata (senza cioè modifiche formali al testo della Carta) ed anche (b.2) in un quadro di continuità nell'interpretazione e nell'applicazione pratica delle regole costituzionali vigenti.

Questa constatazione, nel dibattito politico - ma anche dottrinale - di almeno un ventennio dal 1993, ha indotto molti sostenitori del modello maggioritario ad individuare nella Costituzione del 1948 la principale 
"responsabile" della mancata realizzazione (piena) di questo modello in Italia. Di qui le numerose proposte di revisione - talvolta radicale - del testo costituzionale che sono state presentate ${ }^{137}$, tutte volte a "stabilizzare" il parlamentarismo maggioritario (costituzionalizzandolo, appunto) ${ }^{138}$. Si tratta di proposte basate, inoltre, sul convincimento (a cui è sotteso un giudizio di valore) per cui solo il sistema maggioritario garantirebbe la stabilità e l'"efficienza decisionale" dei governi e sarebbe pertanto «indispensabile» in Italia, sul piano istituzionale, per affrontare in modo efficace i gravi problemi esistenti ${ }^{139}$. In realtà - al di là di ogni valutazione sulla fondatezza di quest'ultima asserzione, evidentemente opinabile -, l'incompiutezza della "transizione" italiana dopo il 1993 (sulla quale invece non possono nutrirsi seri dubbi ${ }^{140}$ ) deve imputarsi principalmente a fattori diversi dalle regole costituzionali: nello specifico, alla struttura ed alle dinamiche del sistema politico, che nel periodo in questione si sono rivelate refrattarie all'instaurazione di un parlamentarismo maggioritario compiuto. Come si è visto sopra, gli attori politici non sono stati capaci (o non hanno voluto), dopo lo sconvolgimento del sistema politico derivante dalle inchieste giudiziarie del 1992/1993 e la riforma della legge elettorale imposta dal referendum sempre del 1993, assumere dei comportamenti pratici coerenti con il modello maggioritario tipico dei parlamentarismi stranieri (quello inglese in primo luogo). Ed anzi nel periodo più recente, in particolare - inaugurato dalle elezioni politiche del 2013 e che sta proseguendo nella legislatura attualmente in corso -, hanno evidenziato delle dinamiche tipiche della fase anteriore al 1993, connotata dal «multipartitismo esasperato» e da un parlamentarismo tipicamente «compromissorio ${ }^{141_{»}}$.

Invero, la Costituzione repubblicana in tema di forma di governo è un testo strutturalmente «aperto», e perciò compatibile con forme (o «varianti») diverse di parlamentarismo ${ }^{142}$. La realizzazione pratica dell'una o dell'altra forma non dipende quindi solo (né, forse, tanto) dalla struttura e dal contenuto delle disposizioni costituzionali, ma - appunto dai comportamenti pratici dei soggetti della forma di governo stessa: cioè innanzitutto i partiti. Se tali comportamenti non si indirizzano in modo stabile e sufficientemente condiviso verso un determinato assetto della forma di governo, questo non può realizzarsi in concreto, nonostante la 
presenza di regole giuridiche ad esso favorevoli (o, almeno, coerenti con esso). Ciò testimonia il rapporto - problematico - di necessaria «interazione» che lega le regole giuridiche ed $\mathrm{i}$ «fatti politici» (e, in particolare, la prassi degli organi costituzionali) ai fini della determinazione di una forma di governo storica, del quale si è detto all'inizio di questo scritto.

\section{UN PERIODO CONTRASSEGNATO DA PROPOSTE DI REVISIONE COSTITUZIONALE SULLA FORMA DI GOVERNO}

Non è possibile concludere un'analisi dedicata all'evoluzione degli orientamenti della forma di governo in Italia senza fare almeno un cenno alle proposte ed ai tentativi di riforma delle disposizioni costituzionali sulla forma di governo stessa, che sono stati effettuati nel periodo successivo al 1993. Essi infatti, per il loro numero e contenuto (oltre che per l'importanza che hanno assunto nel dibattito politico e della dottrina costituzionalistica), rappresentano una delle "cifre" caratterizzanti questa intera fase di storia della repubblica.

Tali progetti, come si è già detto, muovono tutti da un giudizio opinabile, ma largamente condiviso dagli attori politici - di "inadeguatezza sopravvenuta" delle regole costituzionali sulla forma di governo e, in particolare, sui rapporti tra il parlamento ed il governo: queste regole vengono giudicate inadeguate rispetto alle esigenze di stabilità dell'esecutivo e di rapidità ed "efficienza decisionale" dello stesso. Dall'altro lato (e questo è un altro dato rilevante per la presente analisi), le proposte in questione sono accomunate dal fatto di essere fallite in concreto, poiché nessuna di esse è giunta ad approvazione compiendo tutti i passaggi previsti dall'art. 138 della Costituzione (compresa quella, di grande estensione, elaborata dalla maggioranza di centrosinistra nell'ultima legislatura - d.d.l. costituzionale Renzi- Boschi -, respinta dal referendum del 4 dicembre $2016^{143}$ ); e ciò per ragioni diverse ${ }^{144}$.

Ci si limita, in questa sede, ad elencare i più importanti progetti di riforma costituzionale - onde appunto evidenziarne il numero, oltre che 
gli estremi di riferimento -, rinviando a trattazioni più approfondite per la loro specifica disamina ${ }^{145}$ :

-1) progetto della Commissione parlamentare per le riforme istituzionali costituita nella XI legislatura (c.d. «Commissione De Mita Iotti»), presentato ai presidenti delle due camere l'11 gennaio $1994^{146}$;

-2) progetto del Comitato di studio sulle riforme istituzionali, elettorali e costituzionali nominato nella XII legislatura (c.d. «Comitato Speroni»), presentato al governo Berlusconi I il 21 dicembre 1994147;

-3) progetto della Commissione parlamentare per le riforme costituzionali - c.d. «Bicamerale D’Alema» (XIII legislatura, 1997 - 1998), istituita dalla legge costituzionale n. 1/1997148;

-4) d.d.l. costituzionale del governo Berlusconi II (XIV legislatura), approvato definitivamente (a maggioranza assoluta) il 16 novembre 2005, ma respinto dal referendum ex art. 138 Cost. svoltosi il 25 - 26 giugno 2006;

-5) proposta di legge costituzionale approvata dalla Commissione Affari costituzionali della camera il 17 ottobre 2007 (c.d. «Bozza Violante», XV legislatura) ${ }^{149}$;

-6) relazione finale del Gruppo di lavoro sulle riforme istituzionali nominato dal presidente della repubblica il 30 marzo 2013 (all'inizio della XVII legislatura: cc.dd. «saggi di Napolitano») ${ }^{150}$;

-7) relazione finale della Commissione per le riforme costituzionali istituita dal presidente del consiglio l'11 giugno 2013 (c.d. «Commissione dei 35 saggi», XVII legislatura, 12 giugno - 17 settembre 2013) ${ }^{151}$;

-8) d.d.l. costituzionale del governo Renzi (d.d.l. cost. «Renzi-Boschi», XVII legislatura), approvato definitivamente (a maggioranza assoluta) l'11 gennaio 2016, ma respinto dal referendum ex art. 138 Cost. svoltosi il 4 dicembre $2016^{152}$.

Dalla mancata approvazione di questi - numerosi - progetti deriva l'immutatezza/continuità (o se si preferisce, la "resistenza") delle regole costituzionali sulla forma di governo, a cui si è fatto riferimento in precedenza. 


\section{Note}

1 Il quale rappresenta il protagonista politico principale di almeno un ventennio di storia repubblicana italiana, dal 1993 al 2013.

2 Insieme ad altri fattori di cui non si intende certo diminuire la rilevanza: in particolare, le inchieste giudiziarie note come «Tangentopoli»; la crisi monetaria del 1992; le stragi di mafia; e - nel contesto sovranazionale - la fine della c.d. «guerra fredda» e della contrapposizione degli Stati in due «blocchi» (v., in proposito, P. Ginsborg, L'Italia del tempo presente. Famiglia, società civile, Stato. 1980 - 1996, Torino, Einaudi, 1998, pp. 481 ss.).

3 A questo preciso snodo - politico e giuridico - fanno riferimento espressioni come «rivoluzione costituzionale» (C. Fusaro, La rivoluzione costituzionale, Soveria Mannelli, Rubbettino, 1993) o le più comuni «Prima» e «Seconda Repubblica», utilizzate dai media - ma pure, in parte, dalla riflessione costituzionalistica - dopo il 1993 (G.U. Rescigno, A proposito di Prima e Seconda Repubblica, in «Studi parlamentari e di politica costituzionale», 1994, n. 1, pp. 5 ss.; M. Ainis, La forma di governo della Terza Repubblica, in «Quaderni costituzionali», 2019, n. 1, pp. 111 ss., che ha parlato anche, appunto, di «Terza Repubblica», con riferimento alla fase politico- costituzionale apertasi dal 2013, di cui subito infra nel testo).

4 Cfr. ancora, al riguardo, M. Ainis, op. ult. cit., per il quale «al 2013 risale ... la nuova geografia politica italiana ...: tre grandi minoranze, armate l'una contro l'altra. Destra, sinistra, 5 Stelle, separate da pochi punti percentuali. Dunque un sistema tripolare»; A. Spadaro, L'evoluzione della forma di governo. Dal parlamentarismo rigido e razionalizzato al parlamentarismo flessibile, con supplenza presidenziale, in «Quaderni costituzionali», 2019, n. 1, p. 85, che divide la storia repubblicana italiana in tre fasi «caratterizzate dal mutamento [della] legge elettorale e [del] sistema dei partiti»: 1948-1992; 1993-2017; 2018-oggi; nonché S. Cassese, Con il governo è cambiata anche la forma di governo?, in «Quaderni costituzionali», 2019, n. 1, p. 117.

5 Legge n. 165/2017, nota in Italia come «Rosatellum» dal nome del parlamentare proponente, on. Rosato.

6 Si tratta, precisamente, di 193 seggi al Senato e di 386 seggi alla Camera, corrispondenti - per entrambe le Camere - a circa due terzi dei seggi totali.

7 Richiamando una nota definizione di Leopoldo Elia (in Forme di Stato e forme di governo, in S. Cassese (a cura di), Dizionario di diritto pubblico, Milano, Giuffrè, 2006, p. 2603), sulla quale si tornerà tra breve.

8 In due anni dallo svolgimento delle elezioni si sono già succeduti due Governi, sostenuti da due maggioranze politiche diverse (presieduti invece, peraltro - in modo insolito a fronte di questo dato politico - dallo stesso Presidente del Consiglio, Giuseppe Conte).

9 V., in particolare, B. Caravita, I Governi Conte: aspetti problematici di diritto costituzionale, in «Osservatorio A.I.C.» (www.osservatorioaic.it), 2019, n. 5, nonché i diversi contributi raccolti in "Quaderni costituzionali», 2019, n. 1 (in parte citati infra), spec. pp. 77 ss.

10 Cfr., tra i molti possibili riferimenti, A. Spadaro, L'evoluzione della forma di governo, cit., p. 90, per il quale oggi l'Italia «è ... ancora in piena transizione, non si sa ancora bene verso dove».

11 C. Mortati, Le forme di governo, Padova, Cedam, 1973 (che riprende alcuni studi precedenti); L. Elia, Governo (forme di), in Enciclopedia del diritto, Milano, Giuffrè, 1970, vol. XIX, pp. 634 ss.; Id., Forme di Stato e forme di governo, cit., p. 2600.

120 «di indirizzo politico»: la sottolineatura dell'afferenza all'«indirizzo politico» delle funzioni e degli organi di cui si discute è una costante nelle definizioni della dottrina (oltre a Mortati ed Elia, v. per es. M. Volpi, Forme di governo, in Glossario di diritto pubblico comparato, a cura di L. Pegoraro, Roma, Carocci, 2009, p. 142; M. Luciani, Governo (forme di), in Enciclopedia del diritto Annali III, Milano, Giuffrè, 2010, p. 540; A. Di Giovine, Le forme di governo, in Diritto costituzionale comparato, a cura di P. Carrozza, A. Di Giovine e G. F. Ferrari, Roma - Bari, Laterza, 2009 ${ }^{1}$, p. 705; G. Amato, Forme di Stato e forme di governo, Bologna, Il Mulino, 2006, p. 15).

13 Più precisamente, secondo l'ultima definizione data da Elia (in Forme di Stato e forme di governo, cit., p. 2600), la forma di governo è il «[modo] in cui si struttura e si esprime l'insieme degli organi di suprema direzione politica dello stato». In senso analogo, A. Di Giovine, Le forme di governo, 
cit., p. 705, secondo cui si tratta delle «regole che presiedono all'allocazione del potere politico fra gli organi costituzionali dello Stato cui compete la decisione dell'indirizzo politico [e dei] rapporti che si instaurano fra di loro».

14 Nella voce Governo (forme di), cit., pp. 634 ss., ripresa - da ultimo - in Forme di Stato e forme di governo, cit., pp. 2601 ss.. Per un'altra utile classificazione, aggiornata agli ultimi sviluppi del diritto comparato, v. A. Di Giovine, Le forme di governo, cit., pp. 707 ss..

15 Secondo Elia, nella forma di governo parlamentare il rapporto tra il potere legislativo e l'esecutivo è di «continuità» (l'esecutivo è «emanazione permanente» del Parlamento, ha osservato questo Autore nella voce enciclopedica del 1970, sopra citata, con un'espressione che ha avuto grande fortuna nella dottrina successiva), mentre nella forma di governo presidenziale è di «rigida separazione»: Forme di Stato e forme di governo, cit., p. 2601.

16 L. Elia, Governo (forme di), cit., pp. 634 ss.; A. Di Giovine, Le forme di governo, cit., p. 728.

17 Al riguardo Elia richiama rispettivamente - quali esperienze costituzionali in cui le tre «varianti» di parlamentarismo si sono concretizzate - l'ordinamento inglese; gli ordinamenti tedesco e spagnolo; quanto infine al «multipartitismo esasperato», la IV repubblica francese (1946/1958) ed il sistema italiano antecedente al 1993 (ed all'avvento del «bipolarismo di investitura»: L. Elia, Forme di Stato e forme di governo, cit., p. 2603).

18 C. Pinelli, Forme di Stato e forme di governo. Corso di diritto costituzionale comparato, Napoli, Jovene, $2009^{2}$, pp. 149 ss.

19 C. Mortati, Le forme di governo. Lezioni, Padova, Cedam, 1973, pp. 145 ss.

20 La forma di governo semipresidenziale della classificazione di Elia (tipica, in particolare, della V Repubblica francese) viene quindi ascritta, qui, ad una «variante dualista» del parlamentarismo.

21 L. Elia, Forme di Stato e forme di governo, cit., p. 2603.

22 Se non - addirittura - ad un'altra tipologia di forma di governo, non qualificabile come «parlamentare»: v., in particolare, oltre il § 3 , in relazione alla tendenza all'emersione in primo piano (specialmente nell'ultima parte del periodo qui considerato) del presidente della repubblica.

23 Da questo punto di vista, la tassonomia di Pinelli risulta meno utile, obiettivamente, per inquadrare e comprendere las mutaciones che di fatto hanno coinvolto la forma di governo in Italia negli ultimi venticinque anni.

24 Il riferimento è a Massimo Luciani e, più precisamente, alla sua voce Governo (forme di), cit., pp. 553 ss. Più di recente v. anche la critica di S. Cassese, Con il governo è cambiata anche la forma di governo?, cit., p. 116.

25 Di recente, peraltro, Mario Dogliani ha proficuamente ricordato (in Id., C'è ancora posto per il diritto costituzionale in tema di forma di governo?, in «Quaderni costituzionali», 2019, n. 1, pp. 119 ss.) che i modelli delle forme di governo, oltre ad essere uno «strumento ottico per analizzare le singole forme di governo storiche, svolgono una funzione ad docendum sia in discorsi di diritto comparato, di scienza politica, di storia delle istituzioni o di filosofia politica, sia in discorsi di diritto positivo volti ad individuare e descrivere la forma di governo statuita dalla Costituzione positiva. In quest'ultimo caso la funzione ad docendum sconfina in quella ad iubendum, in quanto guida 1) la costruzione di un sistema di norme scritte (legandole concettualmente) e 2) la ricerca di - e la attribuzione di validità a - norme consuetudinarie in senso stretto con cui integrare il sistema medesimo (in quanto ad esso concettualmente ricollegabili). Questo sistema di norme, scritte e consuetudinarie, è la forma di governo positiva». Dogliani, al riguardo, si interroga sulla capacità del diritto costituzionale di «conformare» a sé la politica che ne rappresenta l'oggetto -, esprimendo una posizione pessimista.

26 Ibidem, pp. 583 ss. Per quali siano, secondo Luciani, tali «elementi» di analisi, v. poco più avanti nel testo.

27 Nell'esemplare voce pubblicata nel 1970 nell'Enciclopedia del diritto: L. Elia, Governo (forme di), cit., pp. 638 ss.; ma v. anche, successivamente, l'ulteriore sviluppo in Id., Forme di Stato e forme di governo, cit., pp. 2600 ss. Sull'approccio di Elia ai problemi giuridici in generale - definito da egli stesso «realismo critico» - v. M. Dogliani, Leopoldo Elia: dalla verità dei fatti alla verità nella scienza del diritto, in «Diritto pubblico», 2008, n. 3, pp. 899 ss.

28 V., in particolare, L. Paladin, Diritto costituzionale, Padova, Cedam, $1998^{3}$, pp. 67 ss.; G. Silvestri, La separazione dei poteri, Milano, Giuffrè, 1984, tomo II, pp. 270 ss.; S. Mangiameli, La forma di 
governo parlamentare. L'evoluzione nelle esperienze di Regno Unito, Germania e Italia, Torino, Giappichelli, 1998.

29 Questa definizione è stata espressa dall'illustre studioso nel suo ultimo lavoro su questo tema: L. Elia, Forme di Stato e forme di governo, cit., p. 2600. Nella voce Governo (forme di), cit., del 1970 (in particolare, alle pp. 638 - 642) Elia aveva parlato in proposito - anziché di «situazioni condizionanti» - addirittura di «elementi costitutivi» della forma di governo: vi è stata quindi sul punto un'evoluzione del suo pensiero.

30 L. Elia, Governo (forme di), cit., pp. 638 ss. In particolare, per quanto riguarda l'Italia, gli artt. 49, 72 e 82 della Costituzione «presuppongono una pluralità di partiti e di gruppi parlamentari», i quali rappresentano perciò una «condizione di fatto di immediata rilevanza giuridica». Proprio da questo approccio teorico discende, in particolare, la «sottoclassificazione interna» della forma di governo parlamentare - da parte di Elia - nelle tre «varianti» «a bipartitismo rigido», «a multipartitismo temperato» ed «a multipartitismo esasperato», alle quali si è fatto riferimento sopra.

31 M. Luciani, Governo (forme di), cit., pp. 551 ss. e, in particolare, 553 ss.

32 «L'insieme», cioè, «delle regole giuridiche dei rapporti fra i poteri e organi costituzionali titolari di attribuzioni decisionali di natura politica» (che sono "propriamente giuridiche in quanto positivamente vincolanti»): ibidem, p. 540.

33 Intesa, appunto, "in astratto", come «struttura» dei rapporti tra i poteri e gli organi costituzionali di un determinato ordinamento.

34 Secondo Luciani «tutto ciò che sta fuori dalla forma, dal dato specificamente normativo, non può essere un elemento di quella stessa forma, ma deve essere collocato sul piano, diverso, del "sistema politico- istituzionale". Un piano, questo, che ... non è affatto estraneo allo studioso di diritto costituzionale, eppure richiede griglie analitiche diverse da quella approntata per la classificazione delle forme di governo. In definitiva: se la forma di governo come "forma" deve avere ancora un senso, essa può abbracciare solo il dato normativo e deve tenere lontani tutti gli altri» (M. Luciani, Governo (forme di), cit., p. 556). In particolare, non possono essere considerati nell'analisi della forma di governo i partiti e i sindacati (perché non «incorporati» nei poteri ed organi costituzionali di cui all'art. 134 Cost.), che sono invece centrali - come si è visto - nell'impostazione analitica di Elia.

35 M. Luciani, Governo (forme di), cit., p. 566 s.

36 Come peraltro sembra fare la dottrina maggioritaria in Italia, che nell'analisi della forma di governo tende ad "integrare" le due impostazioni: v., in particolare, G. Amato, Forme di Stato e forme di governo, cit., specialmente pp. 22 ss.; A. Di Giovine, Le forme di governo, cit., p. 706 e pp. 716 ss.; R. Cherchi, La forma di governo: dall'Assemblea costituente alle prospettive di revisione costituzionale, in «Costituzionalismo.it» (www.costituzionalismo.it), 2008, n. 3; da ultimo A. Spadaro, L'evoluzione della forma di governo, cit., pp. 77 s.; quanto all'analisi di aspetti specifici della forma di governo, per esempio, A. Ruggeri, Le crisi di governo tra "regole" costituzionali e "regolarità" della politica, in Le crisi di Governo nell'ordinamento e nell'esperienza costituzionale. Atti di un Convegno, Catanzaro 22 e 23 ottobre 1999, a cura di L. Ventura, Torino, Giappichelli, 2001, in particolare pp. 35 ss.; P. Costanzo, La gestione delle crisi di governo e lo scioglimento anticipato delle Camere, in «Consulta on line» (www.giurcost.org/studi/CostanzoMessina.htm), 2010, in particolare $\S \S 7$ - 8; L. Carlassare, Presidente della Repubblica, crisi di governo e scioglimento anticipato delle Camere, in Il Presidente della Repubblica, a cura di M. Luciani e M. Volpi, Bologna, Il Mulino, 1997, pp. 123 ss.; R. Cherchi, Le crisi di Governo tra Costituzione ed effettività, in «Costituzionalismo.it» (www.costituzionalismo.it), 2011, n. 1.

37 In questo senso, per es., A. Di Giovine, Le forme di governo, cit., p. 716, ha osservato che «per offrire la rappresentazione di una forma di governo il più possibile aderente al suo concreto funzionamento, non ci si può limitare a prendere in considerazione i dati giuridici che la disciplinano, ma si deve tenere presente l'“ambiente” in cui essi operano. ... L'osservazione storico - comparatistica insegna che la forma di governo parlamentare è convissuta e/o convive con i più differenti assetti di partito ... e con un'ampia gamma di sistemi elettorali; inoltre in essa sia il Parlamento, sia il Governo, sia il suo vertice possono assumere ruoli e status diversi, e anche il ruolo del Capo dello Stato ... si muove entro una banda di oscillazione piuttosto ampia». 
38 Per Luciani - più precisamente - la «concret[a] esperienz[a] giuridico - politic[a]»: Id., Governo (forme di), cit., p. $566 \mathrm{~s}$.

39 Non vengono invece affrontati direttamente in questa sede i profili attinenti al rapporto tra il sistema dei partiti e le regole elettorali (che pure rivestono, evidentemente, grande importanza), cioè tutto quanto investe il momento elettorale e le fasi e le dinamiche che lo precedono. Tali profili meriterebbero una trattazione autonoma e che esula dai limiti di questo contributo.

40 Per una presentazione efficace delle vicende che hanno interessato i partiti in Italia nel periodo a cavallo del 1993, condotta da un punto di vista storico, si veda P. Ginsborg, L'Italia del tempo presente, cit., pp. 477 ss., in particolare pp. 526 ss.; S. Colarizi e M. Gervasoni, La Tela di Penelope. Storia della Seconda Repubblica, Roma - Bari, Laterza, 2012; La qualità della democrazia in Italia (1992-2012), a cura di L. Morlino, D. Piana e F. Raniolo, Bologna, Il Mulino, 2013.

41 In quanto strettamente collegata alle inchieste giudiziarie note come «Tangentopoli», che - nel giro di pochi mesi - misero a nudo il «sistema» di corruzione e di malaffare presente nei partiti tradizionali fin dai primi anni della vicenda repubblicana e portarono all'arresto ed alla condanna dei loro leaders; e, così, misero a nudo - di fatto - cinquant'anni di storia repubblicana, che ne risultarono a loro volta fortemente screditati (in proposito v. ancora P. Ginsborg, L'Italia del tempo presente, cit., pp. 481 ss., in particolare pp. 501 ss.).

42 Di cui, come si è visto sopra, parla Elia.

43 Pur avendo un consenso elettorale assai elevato. Secondo R. Cherchi, in particolare (La forma di governo, cit., § 3), «tre regolarità informali caratterizzarono il funzionamento della forma di governo sino al 1992: a) il Governo di coalizione, scelta politica della DC nella prima legislatura e "necessità istituzionale" a partire dalla seconda; b) il primato dei partiti sui parlamentari (e sulle delegazioni ministeriali); c) la conventio ad excludendum nei confronti del PCI ... e del MSI».

44 Che, ad uno sguardo retrospettivo, rappresenta - anche per il messaggio che veniva trasmesso da Silvio Berlusconi, circa il suo progetto per la politica ed il governo del Paese - l'autentico momento di passaggio dalla «Prima repubblica» alla fase nuova.

45 Anche se - va ricordato - tale semplificazione si rivelerà, alla prova dei fatti, assai lenta e graduale (la si percepirà soprattutto nella fase terminale del periodo qui in esame, a partire dalla XVI legislatura, 2008/2013) e, peraltro, riguarderà più i gruppi parlamentari (che tenderanno a diminuire, in conseguenza della presentazione delle liste elettorali in coalizioni imposta dalla legislazione elettorale) che non i partiti politici veri e propri. Infatti la proliferazione partitica che aveva contrassegnato, come detto nel testo, la prima parte di storia repubblicana non si arresta dopo il 1993 e, anzi, si accentua in alcuni periodi (per esempio, nella XV legislatura, 2006/2008, con maggioranza politica di centrosinistra): i partiti cambiano natura rispetto al passato, perdendo la loro matrice ideologica e presentandosi spesso come soggetti politici effimeri e "cangianti", non stabilmente collocati all'interno del sistema politico, soprattutto in quanto inscindibilmente legati alla figura di un determinato personaggio politico; ma - proprio in virtù di tali caratteristiche restano numerosi. La legislazione sul finanziamento (pubblico) dei partiti stessi, e dall'altro lato la legislazione elettorale (nonostante la sua impronta complessiva maggioritaria), favoriscono, del resto, la formazione di piccoli gruppi politici, spesso fortemente "condizionanti" le scelte dei partiti più grandi e di tradizione politica più solida, appartenenti alla medesima coalizione (v., a questo proposito, F. Biondi - G. Rivosecchi, Le forme della rappresentanza e il sistema dei partiti, in Vent'anni di Costituzione, cit., pp. 161 ss.).

46 In particolare, per l'impatto del referendum elettorale del 1993 sull'assetto dei partiti in Italia, si rinvia ancora a F. Biondi, op. ult. cit., pp. 170 ss. Si ricordi che, tra le principali ragioni esposte fin dall'inizio da Silvio Berlusconi a sostegno della sua «discesa in campo», vi è proprio quella di evitare la «salita al potere» dei partiti della sinistra.

47 Più precisamente, in occasione delle elezioni politiche del 2001, del 2006, del 2008 ed anche del 2013.

48 V. oltre, $§ 4$.

49 Già sopra citate: precisamente, sentenze n. 1/2014 e n. 35/2017.

50 Prima delle elezioni del 4 marzo 2018 (disciplinate dalla citata legge n. 165/2017, prevalentemente proporzionale) sembrano evidenti la fragilità ed il carattere "estemporaneo" e non solidamente radicato delle coalizioni di centrodestra e centrosinistra, che (insieme al MoVimento 5 Stelle) 
si candidano a governare. Ed infatti, poco dopo le elezioni, la Lega abbandonerà la coalizione di centrodestra (con la quale aveva eletto numerosi parlamentari, in "quota" maggioritaria) e formerà il governo con il MoVimento 5 Stelle.

51 V., in particolare, M. Timiani, Crisi e formazione dei Governi: tendenze nella prassi del maggioritario (1992 - 2007), in La prassi degli organi costituzionali, a cura di A. Barbera e T.F. Giupponi, Bologna, Bononia University Press, 2008, pp. 69 - 100; per il periodo successivo (e a noi più vicino), N. Maccabiani, Il ruolo del presidente della repubblica a fronte delle difficoltà interne alle maggioranze parlamentari di centro- sinistra e di centro- destra durante la XV e la XVI legislatura, in «Rivista A.I.C.» (www.rivistaaic.it), 2011, n. 1. A questo proposito Roberto Cherchi (ne La forma di governo, cit., § 3) osserva che l'instabilità politica e dei governi dopo il 1993 dipende dalla «deistituzionalizzazione dei partiti e [dalla] personalizzazione della politica», che «hanno sensibilmente indebolito la capacità dei partiti di irreggimentare le condotte dei parlamentari (e dei Ministri)».

52 I quali, come già si è ricordato (v. sopra, nota 45), restano complessivamente numerosi nel corso del ventennio qui esaminato (anche all'interno delle coalizioni politiche), perdono la loro caratterizzazione ideologica e tendono a divenire soggetti politici effimeri e "cangianti", non stabilmente collocati nel sistema, soprattutto in quanto legati ad un determinato personaggio politico ed alle sue alterne fortune (secondo il modello del c.d. «partito personale»).

53 Il parlamentarismo maggioritario, del resto - in Inghilterra così come in altri contesti in cui esso si è realizzato -, vede contrapporsi di norma due principali partiti (pur eterogenei nella loro composizione interna), anziché coalizioni (cfr. A. Di Giovine, Le forme di governo, cit., pp. 717 e 720). In Italia, viceversa - come già accennato -, la tendenza alla semplificazione del sistema politico evidenziata nel testo non ha prodotto, lungo il ventennio 1993/2013, un sistema bipartitico, dal momento che sono rimasti in vita numerosi partiti (alcuni dei quali ostili all'evoluzione maggioritaria).

54 Specialmente i partiti più piccoli dei due principali schieramenti politici.

55 Sui delicati - e, a loro volta, conflittuali - rapporti, invece, tra il «circuito» maggioranza parlamentare - governo da un lato e gli organi di garanzia dall'altro lato (in primo luogo, il presidente della repubblica), v. oltre, §§ 2.4 e 3.

56 Questi profili, a loro volta, risultano strettamente legati al sistema dei partiti, innanzi esaminato: in una forma di governo parlamentare sono i comportamenti dei partiti politici, e le loro vicende interne, a determinare primariamente la nascita e la permanenza in carica degli esecutivi.

57 M. Timiani, Crisi e formazione dei Governi, cit., pp. 70 ss.; G. Pitruzzella, Artt. 92 - 93, in Commentario della Costituzione. Art. 92 - 96, a cura di G. Branca e A. Pizzorusso, Bologna, Zanichelli, 1994, pp. 49 ss.; G. Rivosecchi, Fiducia parlamentare, in Digesto delle discipline pubblicistiche. III Aggiornamento, Torino, Utet, 2008, vol. I, spec. pp. 386 ss.; M. Midiri, Fiducia parlamentare, in Dizionario di diritto pubblico, a cura di S. Cassese, Milano, Giuffrè, 2006, vol. III, pp. 2489 ss.

58 Ma anche quello (più frequente nella prassi) che porta dalla crisi di un governo alla nomina di un altro governo durante il corso della legislatura, senza che si giunga allo scioglimento anticipato delle camere.

59 Il primo governo presieduto da Conte si è formato dopo la conclusione di un inedito - e assai problematico dal punto di vista dell'inquadramento (e della sua stessa legittimità) costituzionale - «contratto di governo» tra le due forze politiche componenti la maggioranza, che si erano presentate contrapposte alle elezioni del 4 marzo 2018: il MoVimento 5 Stelle e la Lega. In proposito v. le considerazioni critiche di M. Cavino, The Manchurian Candidate. Il Presidente del Consiglio garante del contratto di governo, nel fascicolo monografico di «federalismi.it» (www.federalismi. it), 2019, n. 3 (numero speciale), a cura di M. Malvicini, Parlamento e governo parlamentare in Italia. Verso un affresco contemporaneo, pp. 231 ss.

60 Sul nesso di strumentalità tra il ricorso frequente al voto di fiducia ed il «rafforzamento della posizione direttiva dell'Esecutivo verso la "sua" maggioranza», a fronte di difficoltà del rapporto fiduciario nel suo complesso, v. G. Rivosecchi, Fiducia parlamentare, cit., pp. 396 ss..

$61 \mathrm{~V}$.i dati analiticamente riportati, per ciascuna legislatura, nel sito istituzionale del parlamento (al link http://www.senato.it/leg/17/BGT/Schede/Statistiche/Stato//DDLQuestioneFiducia.html) e 
nei Rapporti sullo stato della legislazione pubblicati dalla Camera dei deputati, Osservatorio sulla legislazione, dal 1998 in avanti (reperibili al link http://leg16.camera.it/397?documenti=168).

62 Tanto che la prassi in questione è stata oggetto di censure e richiami sempre più frequenti da parte del presidente della repubblica. Più precisamente, nella XIV legislatura $(2001 / 2006)$ i governi hanno posto la fiducia, complessivamente, su 16 d.d.l. al senato e 25 d.d.l. alla camera; nella XV legislatura (durata solo due anni: 2006/2008) su 10 al senato e 12 alla camera; nella XVI legislatura (2008/2013) su 31 al senato e 48 alla camera; nella XVII legislatura (2013/2018) addirittura su 56 al senato e 44 alla camera.

63 Naturalmente, in circostanze diverse da quelle in cui l'esecutivo ha posto la fiducia (nei termini appena visti): l'ordinamento italiano prevede infatti che, qualora le Camere non approvino le proposte sulle quali il governo ha posto formalmente la fiducia - mediante un'apposita dichiarazione -, il governo stesso sia obbligato a dimettersi.

64 Cfr., in dottrina, L. Paladin, Governo italiano, in Enciclopedia del diritto, Milano, Giuffrè, 1970, vol. XIX, pp. 697 s., secondo cui il disposto dell'art. 94 Cost. non consente «separazioni precise tra le responsabilità collegiali ed individuali dei membri del Governo». Nello stesso senso, G.U. Rescigno, La responsabilità politica, Milano, Giuffrè, 1967, pp. $236 \mathrm{~s}$.

65 Riguardante il ministro della giustizia del governo Dini Filippo Mancuso, colpito da mozione di sfiducia individuale approvata dal Senato il 19 ottobre 1995, a cui ha fatto seguito il conferimento dell'incarico di ministro, ad interim, al presidente del consiglio, operato dal presidente della Repubblica lo stesso giorno.

66 Inquadrandolo, come è noto, nella «struttura» generale della forma di governo parlamentare delineata dalla Costituzione repubblicana: punti 8 e 12 del «Considerato in diritto» della sentenza.

67 Sull'istituto della mozione di sfiducia individuale v., per es., F. Donati, La responsabilità politica dei ministri nella forma di governo italiana, Torino, Giappichelli, 1997; G. Rivosecchi, Fiducia parlamentare, cit., pp. 394 ss.; L. Carlassare, Sfiducia individuale e revoca dei ministri nel nuovo assetto politico italiano, in Aa. Vv., Scritti in onore di Serio Galeotti, Milano, Giuffrè, 1998, vol. I, pp. 157 ss.

68 Ed anche, correlativamente - come si vedrà di seguito -, al non esercizio.

69 Sulle crisi di governo e lo scioglimento anticipato delle camere si segnalano (nell'ambito di una bibliografia vastissima) A. Ruggeri, Le crisi di governo tra "regole" costituzionali e "regolarità" della politica, cit., pp. 34 ss.; S. Bartole, Scioglimento delle Camere, in Enciclopedia del diritto. Aggiornamento, Milano, Giuffrè, 1999, vol. III, pp. 936 ss.; T.E. Frosini e P.L. Pietrillo, Art. 88, in Commentario alla Costituzione, a cura di R. Bifulco, A. Celotto e M. Olivetti, Torino, Utet, 2006, vol. II, pp. 1712 ss.; R. Cherchi, Le crisi di governo tra Costituzione ed effettività, cit.; R. Cherchi, Lo scioglimento delle Camere nella Costituzione italiana, in «Costituzionalismo.it» (www.costituzionalismo.it), 2012, n. 2; L. Carlassare, Presidente della Repubblica, crisi di governo e scioglimento delle Camere, cit.; P. Costanzo, La gestione delle crisi di governo e lo scioglimento anticipato delle Camere, cit.; A. Ruggeri, Crisi di governo, scioglimento delle Camere e teoria della Costituzione, in Evoluzione del sistema politico - istituzionale e ruolo del Presidente della Repubblica: atti di un incontro di studio (Messina - Siracusa, 19 e 20 novembre 2010), a cura di A. Ruggeri, Torino, Giappichelli, 2011, pp. 465 ss.

70 Va rilevato, peraltro, che queste due crisi sono anche le uniche di natura "parlamentare» dell'intera storia repubblicana in Italia: il fatto che esse si situino nel periodo dopo il 1993 può essere spiegato, forse (almeno per quanto riguarda la seconda, del 2008), con lo sviluppo della tendenza alla c.d. «parlamentarizzazione delle crisi», di cui subito oltre nel testo.

E' opportuno inoltre ricordare che da ultimo, con riferimento alle vicende che nell'estate del 2019 hanno determinato le dimissioni del governo Conte I (formato dopo le elezioni politiche del 4 marzo 2018 e sostenuto dal MoVimento 5 Stelle e dalla Lega), in dottrina è stata coniata l'espressione (/categoria "intermedia") «crisi di governo semi-parlamentare»: ciò in quanto, da una parte, la crisi è maturata in seguito alla presentazione di una mozione di sfiducia contro il governo da parte di una delle forze politiche che lo sostenevano (la Lega di Salvini), ed è stata oggetto di un ampio dibattito in parlamento, avvenuto il 20 agosto 2019 (e che, per i suoi contenuti, ha avuto una grande risonanza presso l'opinione pubblica); dall'altra parte, tuttavia, non vi è stato un vero e proprio voto delle camere sulla mozione di sfiducia, ed il presidente Conte ha 
rassegnato le dimissioni subito dopo la citata seduta del 20 agosto, dedicata, formalmente, alle "comunicazioni" del presidente del consiglio sulla situazione politica (v., al riguardo, B. Caravita, I Governi Conte, cit., p. 4).

71 M. Timiani, Crisi e formazione dei Governi, cit., p. 80.

72 Sulla quale v., in particolare, P. Costanzo, La gestione delle crisi di governo e lo scioglimento anticipato delle Camere, cit., § 2; A. Ruggeri, Le crisi di governo tra "regole" costituzionali e "regolarità" della politica, cit., pp. 87 ss..

73 Essa consiste, secondo la prassi del periodo temporale qui esaminato, nella richiesta da parte del governo del voto favorevole delle camere su un determinato provvedimento (anche solo una mozione), senza l'apposizione della questione di fiducia, ma attribuendo comunque al voto parlamentare un «significato fiduciario» sul piano politico: M. Timiani, Crisi e formazione dei Governi, cit., p. 84 (dove anche alcuni esempi concreti tratti dal periodo in esame).

74 Questo specifico profilo rappresenta, peraltro, una sostanziale novità nell'esperienza politicoistituzionale italiana.

75 Come proprio, da ultimo, la crisi del governo Conte I, innescata dalla decisione della Lega di Salvini di revocare la fiducia all'esecutivo: la soluzione della crisi che si è poi determinata - nomina del governo Conte II, sostenuto dal MoVimento 5 Stelle (già partito di maggioranza relativa) e dal Partito democratico (prima all'opposizione) - non era affatto prevedibile e ha "sorpreso" molti analisti ed esponenti politici, a partire dallo stesso Salvini.

76 S. Bartole, Scioglimento delle Camere, cit., spec. pp. 939 ss.; A. Ruggeri, Evoluzione del sistema politico - istituzionale e ruolo del Presidente della Repubblica: notazioni introduttive, in Evoluzione del sistema politico - istituzionale e ruolo del Presidente della Repubblica, cit., pp. 3 ss.; N. Maccabiani, Il ruolo del presidente della repubblica a fronte delle difficoltà interne alle maggioranze parlamentari, cit. Per quanto attiene, nello specifico, alle due presidenze Napolitano, pur essendo prematuro esprimere giudizi definitivi, pare possibile rilevare un'evoluzione tra di esse proprio in relazione alla gestione delle crisi di governo ed all'interpretazione del potere di scioglimento: da un approccio tradizionale, volto a "registrare" gli orientamenti delle forze politiche e a sciogliere le camere in caso di mancato accordo tra queste (e quindi, di richiesta dello scioglimento), il presidente Napolitano pare essere passato, negli anni più recenti della sua presidenza, ad attuare la dottrina dello scioglimento come «extrema ratio», da evitare ad ogni costo (anche se del caso "imponendo" ai partiti determinate soluzioni ed alleanze: si pensi alla nomina del governo Monti o all'impulso dato alla nascita del governo Letta dopo l'incerto risultato elettorale del 2013, contro le richieste di scioglimento di molti esponenti politici).

77 Per una disamina puntuale delle crisi di governo di questo periodo e dei loro rispettivi svolgimenti ed esiti, v., oltre ai lavori citati alla nota 69, M. Timiani, Crisi e formazione dei Governi, cit., pp. 80 ss. (dove anche un prospetto cronologico dettagliato fino al 2007); S. Leone, Il potere di scioglimento anticipato delle Camere: teoria e prassi in un contesto istituzionale in trasformazione, in «Quaderni regionali», 2011, n. 1, pp. 177 ss.; C. De Girolamo, Lo scioglimento anticipato delle Camere: una ricognizione della dottrina e della prassi, in «Forum di Quaderni Costituzionali» (www.forumcostituzionale.it), 5/9/2011.

78 A rigore, oltre agli scioglimenti del 1994, del 1996 e del 2008 di cui subito si dirà nel testo, in altre tre occasioni le camere sono state sciolte in anticipo rispetto alla scadenza naturale della legislatura: nel 2001, nel 2006 e nel 2013. Ma nei primi due casi, ciò è avvenuto per ragioni strettamente tecniche - evitare, nel 2001, lo svolgimento delle elezioni politiche in estate e, nel 2006, la sovrapposizione delle elezioni stesse con l'elezione del nuovo presidente della repubblica (v. P. Costanzo, La gestione delle crisi di governo e lo scioglimento anticipato delle Camere, cit., tabella alla nota 28) -, mentre nel 2013 a causa delle dimissioni del Presidente del consiglio Monti, rassegnate il 21 dicembre 2012, poco tempo prima della scadenza della legislatura e a sessione di bilancio ormai conclusa (v. al riguardo A. Zito, Alla fine della XVI Legislatura: la crisi del governo Monti e lo scioglimento anticipato delle Camere, in «Rassegna Parlamentare», 2013, pp. 163 ss.).

79 Cioè imputabili all'esclusiva volontà del presidente della Repubblica (contro la volontà delle forze politiche). Il decreto di scioglimento del 16 gennaio 1994 viene motivato dal presidente Scalfaro con il cambiamento intervenuto nel sistema elettorale dopo il referendum della primavera del 
1993; con l'approssimarsi delle elezioni amministrative; ma, soprattutto, con la delegittimazione e la «non rappresentatività» del Parlamento in carica in conseguenza delle inchieste giudiziarie di «Tangentopoli» (già citate): secondo il presidente le «varie patologie manifestatesi nella gestione della cosa pubblica» avrebbero "spezzato" il rapporto di fiducia tra gli elettori ed i loro rappresentanti in carica.

80 Quali, in particolare, quelle di P. Costanzo, La gestione delle crisi di governo e lo scioglimento anticipato delle Camere, cit., § 4; L. Carlassare, Presidente della Repubblica, crisi di governo e scioglimento delle Camere, cit., pp. 136 ss.; R. Cherchi, Lo scioglimento delle Camere nella Costituzione italiana, cit., § 3 .

81 Sullo scioglimento «di funzionalità»e, più in generale, sulle classificazioni dottrinarie degli scioglimenti anticipati, v. in particolare A. Mastropaolo, Le ricostruzioni dell'esercizio del potere di scioglimento anticipato in dottrina nell'esperienza repubblicana, in «Archivio di Diritto e Storia Costituzionali» (www.dircost.unito.it), 2012; P. Costanzo, La gestione delle crisi di governo e lo scioglimento anticipato delle Camere, cit., § 3; R. Cherchi, Lo scioglimento delle Camere nella Costituzione italiana, cit., spec. $\S \S 3-7$ - 8; C. De Girolamo, Lo scioglimento anticipato delle Camere, cit., pp. 2 ss.

82 Sul punto v. N. Maccabiani, M. Frau ed E. Tira, Dalla crisi del IV governo Berlusconi alla formazione del I governo Monti, cit., pp. 41 ss.

83 Ma non votata in concreto dalle camere: v. retro, nota 66.

84 E del conseguente mutato quadro politico, almeno fino al 2013/2018: v. retro, § 2.1 .

85 Per riferimenti su questa opinione, nella dottrina, v. S. Bartole, Scioglimento delle Camere, cit., p. 938, nonché A. Spadaro, Poteri del Capo dello Stato, forma di governo parlamentare e rischio di "ribaltone", in «Forum di Quaderni Costituzionali» (www.forumcostituzionale.it), 28/9/2010, ora anche in Studi in onore di Franco Modugno, cit., vol. IV, pp. 3433 ss.; T.E. Frosini e P.L. Petrillo, Verso una interpretazione maggioritaria del potere di scioglimento delle Camere, in «Diritto e società», 2005, n. 2, pp. 137 ss.; S. Leone, Il potere di scioglimento anticipato delle Camere, cit., p. 179 e relativa nota 11 .

86 In proposito si è osservato, in dottrina, che «il Presidente ... ha, sì, il dovere di prendere atto di una maggioranza parlamentare idonea a sostenere un governo, ma anche - e oggi sempre più - quello di accertarsi che tale maggioranza non sia in contrasto con quella espressa dal corpo elettorale o comunque che non ci sia una "frattura" consolidata (per esempio attraverso diverse elezioni e prove referendarie) e inaccettabile (per l'evidenza dell'opinione pubblica) fra la maggioranza parlamentare e l'orientamento prevalente del Paese» (A. Spadaro, Poteri del Capo dello Stato, forma di governo parlamentare e rischio di "ribaltone", cit., p. 13). Più cauto sull'evoluzione della prassi del periodo dopo il 1993, invece, P. Costanzo, La gestione delle crisi di governo e lo scioglimento anticipato delle Camere, cit., § 7 (il quale si limita a sottolineare il «maggior attivismo del Capo dello Stato» nella gestione delle crisi).

87 L. Carlassare, Nel segno della Costituzione. La nostra carta per il futuro, Milano, Feltrinelli, 2012, pp. 172 ss. (in relazione alle riforme del sistema elettorale); da ultimo S. Cassese, Con il governo è cambiata anche la forma di governo?, cit., pp. 116 ss.; in prospettiva comparata, A. Di Giovine, Le forme di governo, cit., p. 721.

88 Che, come si è visto nei precedenti paragrafi, agevolano di fatto la formazione e la permanenza in carica/stabilità del governo stesso.

89 T. Martines, Indirizzo politico, in Enciclopedia del Diritto, Milano, Giuffrè, 1971, vol. XXI, pp. 134 ss.; M. Dogliani, Indirizzo politico, in Digesto delle discipline pubblicistiche, Torino, Utet, 1993, vol. VIII, pp. 244 ss..

90 A. Mastropaolo e L. Verzichelli, Il Parlamento. Le assemblee legislative nelle democrazie contemporanee, Roma - Bari - Laterza, 2006, in particolare cap. 5; G. Rivosecchi, Governo, maggioranza e opposizione a quarant'anni dai regolamenti parlamentari del 1971: dai (presunti) riflessi della stagione consociativa al (presumibile) declino del Parlamento, in «Rivista A.I.C.» (www.rivistaaic.it), 2012, n. 2, e (con il titolo A quarant'anni dal 1971: dai (presunti) riflessi della stagione consociativa al (presumibile) declino del Parlamento) anche in I regolamenti parlamentari a quarant'anni dal 1971, a cura di A. Manzella, Bologna, Il Mulino, 2012, pp. 155 ss.; C. Chimenti, Declino delle Camere nel parlamentarismo maggioritario italiano, in «Queste istituzioni» (www.questeistituzioni.it), 
2004, n. 133 - 134, pp. 49 ss.; in termini critici sull'espressione, A. Barbera, Parlamento, in Enciclopedia delle scienze sociali, Roma, Istituto della enciclopedia italiana Treccani, 1996, vol. VI, pp. 472 ss. (pubblicato, inoltre, come volume con il titolo I Parlamenti: un'analisi comparativa, Roma - Bari, Laterza, 1999), e C. Fusaro, Il declino del Parlamento tra mito e realtà: il caso italiano, in Quaderni dell'Associazione per gli Studi e le Ricerche Parlamentari, Torino, Giappichelli, 2007, n. 18, pp. 29 ss. Da ultimo sul ruolo del parlamento nella forma di governo italiana, sotto vari profili d'indagine, si veda il già citato fascicolo monografico di «federalismi.it» (www.federalismi. it), 2019, n. 3 (numero speciale), a cura di M. Malvicini, Parlamento e governo parlamentare in Italia. Verso un affresco contemporaneo.

91 I rilievi che seguono sono tratti, come appena accennato nel testo, dall'analisi dei dati statistici relativi all'attività legislativa, raccolti e pubblicati ogni anno, ormai, da diverse fonti: i Rapporti sullo stato della legislazione dell'Osservatorio sulla legislazione della Camera dei deputati (dal 1998 in poi), reperibili al link http://leg16.camera.it/397?documenti=168; le statistiche parlamentari pubblicate nel sito istituzionale dello stesso parlamento (al link http://www.senato. it/leg/17/BGT/Schede/Statistiche.html); l'Osservatorio sulle fonti, fondato e diretto da Paolo Caretti (ora anche on-line: http://www.osservatoriosullefonti.it, dove anche diversi saggi e lavori di elaborazione e riflessione sulle dinamiche dell'attività legislativa). In tema, da ultimo, v. E. Longo, La funzione legislativa nella XVI e XVII legislatura. Spunti per una indagine a partire dal Rapporto sulla legislazione 2017-2018, in Parlamento e governo parlamentare in Italia, cit., pp. 13 ss., nonché gli altri contributi a pp. 35 ss. del medesimo fascicolo.

92 Si pensi in particolare, al riguardo, alle leggi di delega o all'iniziativa in materia di revisione costituzionale, entrambe ampiamente esercitate dagli esecutivi. Sono di iniziativa del governo, per esempio - e significativamente -, i d.d.l. di revisione costituzionale approvati nel 2005 e nel 2016 dalle camere e respinti dai referendum popolari, rispettivamente, del 25 - 26 giugno 2006 e del 4 dicembre 2016 (v. anche oltre, § 6). Per quanto concerne i dati dell'iniziativa legislativa ordinaria del governo, solo per fare un esempio, nella XVI legislatura (2008/2013) sono stati approvati 304 d.d.l. di iniziativa governativa, contro appena 91 progetti di legge di iniziativa parlamentare (v. il link http://www.senato.it/leg/16/BGT/Schede/Statistiche.html).

93 V., in proposito, G. Rivosecchi, Governo, maggioranza e opposizione a quarant'anni dai regolamenti parlamentari del 1971, cit., p. 7 (dove gli esatti riferimenti normativi).

94 Solo per citare alcuni esempi nel corso degli anni, le riforme del pubblico impiego e delle pensioni del 1993/1995 (d.lgs. n. 29/1993, legge n. 335/1995, c.d. «riforma Dini»); riforme dell'Università e del lavoro privato del 2010/2012 (legge n. 240/2010, c.d. «legge Gelmini», leggi n. 183/2010 e n. $92 / 2012$, c.d. «collegato lavoro» del governo Berlusconi IV e la c.d. «legge Fornero» - governo Monti); riforme della scuola e, ancora, del lavoro della legislatura 2013/2018 (legge n. 107/2015, sulla «buona scuola», legge n. 183/2014 e relativi d.lgs. di attuazione, c.d. «Jobs Act»).

95 Nel senso che il governo, mentre presenta alla camera il «maxi- emendamento» (che spesso è il frutto di un accordo in sede extra-parlamentare tra i leader dei partiti della maggioranza), pone la fiducia sul testo del provvedimento in discussione, quale risultante dal «maxi- emendamento» stesso: in questo modo, "tronca" sul nascere la discussione parlamentare del testo (F. Biondi - S. Leone, Il Governo "in" Parlamento, cit., pp. 20 ss., che rilevano come questa prassi venga seguita soprattutto nel procedimento di conversione dei decreti- legge; G. Rivosecchi, Governo, maggioranza e opposizione a quarant'anni dai regolamenti parlamentari del 1971, cit., pp. 17 ss.).

96 Sulle carenze dei regolamenti parlamentari ed il dibattito (prevalentemente dottrinario) sulla loro eventuale riforma, v. ancora l'approfondita analisi di F. Biondi - S. Leone, Il Governo "in" Parlamento, cit., spec. pp. 9 ss., nonché N. Lupo, Il procedimento legislativo in Assemblea, nel rapporto (ancora irrisolto) con il Governo, in «Il Filangieri», 2007, pp. 125 ss.

97 Su questa tendenza generale v., tra i tanti, F. Biondi - S. Leone, Il Governo "in" Parlamento, cit., pp. 12 ss.; N. Lupo, Gli atti normativi del Governo tra legalità costituzionale e fuga dai controlli, in Gli atti normativi del Governo tra Corte costituzionale e giudici. Atti del Convegno annuale dell'Associazione "Gruppo di Pisa», a cura di M. Cartabia, E. Lamarque e P. Tanzarella,Torino, Giappichelli, 2011, pp. 535 ss.; e, in una prospettiva di più ampio respiro, A. Pizzorusso, L'ampliamento dei poteri normativi dell'esecutivo nei principali ordinamenti occidentali: a cinquant'anni dal saggio di Enzo Cheli, in Lo Stato costituzionale. La dimensione nazionale e la prospettiva internazionale. Scritti 
in onore di Enzo Cheli, a cura di P. Caretti e M.C. Grisolia, Bologna, Il Mulino, 2010, p. 105 s. (che riflette sul saggio di E. Cheli, L'ampliamento dei poteri normativi dell'esecutivo nei principali ordinamenti occidentali, in «Rivista trimestrale di diritto pubblico», 1959, pp. 463 ss.).

98 Si sofferma su questo profilo, ricavandone delle conclusioni generali che investono la qualificazione della forma di governo tout court, A. Spadaro, L'evoluzione della forma di governo, cit., pp. 91 ss.

99 Che in almeno due occasioni, hanno riguardato leggi di conversione di decreti- legge: si tratta, precisamente, del messaggio alle camere del presidente Ciampi del 29 marzo 2002, avente ad oggetto la legge di conversione del d.l. n. 4/2002 recante «Disposizioni urgenti finalizzate a superare lo stato di crisi per il settore zootecnico, per la pesca e per l'agricoltura»; della lettera ai presidenti delle camere del presidente Napolitano del 27 dicembre 2013, avente ad oggetto la legge di conversione del d.l. n. 126/2013, c.d. «decreto salva- Roma» (in quest'ultimo caso non si è trattato - per il vero di un rinvio vero e proprio, ma ha avuto lo stesso effetto, dal momento che il governo ha poi ritirato la parte del d.d.l. di conversione oggetto delle censure del presidente della repubblica). In entrambi questi casi, il presidente della repubblica ha censurato l'inserimento nel testo del decreto- legge, durante l'iter di conversione, di disposizioni «non omogenee» rispetto al contenuto del decreto né giustificate dai requisiti di necessità e urgenza previsti dall'art. 77 Cost.

${ }^{100}$ Si tratta del rifiuto, da parte del presidente Napolitano, di emanare il decreto- legge approvato il 6 febbraio 2009 dal governo Berlusconi IV, sul divieto di interruzione delle cure alle persone in stato vegetativo permanente (meglio noto come «decreto Englaro», dalla vicenda da cui esso traeva origine): v. al riguardo le riflessioni di A. Spadaro, Può il Presidente della Repubblica rifiutarsi di emanare un decreto-legge? Le "ragioni" di Napolitano, in «Forum di Quaderni Costituzionali» (www.forumcostituzionale.it), 10/2/2009, e di A. Ghiribelli, Il potere del Presidente della Repubblica in sede di emanazione dei decreti - legge: il "caso Englaro", in "Consulta on line» (www.giurcost.org/studi).

${ }^{101}$ Con i quali è stato censurato l'eccessivo ricorso agli strumenti «straordinari» citati nel testo: $\mathrm{v}$., in particolare, le note del 18 maggio 2007,17 aprile 2009, 29 marzo 2012, e le lettere ai presidenti delle camere ed al presidente del consiglio dei ministri del 9 aprile 2009, 22 maggio 2010, 22 febbraio 2011, 7 marzo 2011, 23 febbraio 2012, tutte del presidente Napolitano. Tutti questi documenti sono reperibili nel sito Internet della presidenza della repubblica (www.quirinale. it). Anche su questi messaggi v. F. Biondi - S. Leone, Il Governo "in" Parlamento, cit., pp. 28 ss., e A. Sperti, Il decreto legge tra Corte costituzionale e Presidente della Repubblica dopo la "seconda svolta", in Gli atti normativi del Governo tra Corte costituzionale e giudici ,cit., pp. 3 ss.

${ }^{102}$ La sentenza n. 171/2007 è particolarmente importante, perché per la prima volta la corte costituzionale ha dichiarato costituzionalmente illegittima - ed ha quindi annullato - una disposizione contenuta in un decreto- legge (d.l. n. 80/2004), nonostante l'intervenuta conversione del decreto in legge da parte del parlamento (1. n. 140/2004): v., in particolare, il commento "a prima lettura" di A. Celotto, C'è sempre una prima volta ... (La Corte costituzionale annulla un decreto- legge per mancanza dei presupposti), in «GiustAmm.it» (www.giustamm.it) ed in «Consulta on line» (www. giurcost.org/studi).

103 V. amplius oltre, $\S 4$.

${ }^{104} \mathrm{Nel}$ senso precisato sempre nel § 1 di questo capitolo.

105 Rispettivamente, 1978/1985 e 1985/1992.

${ }^{106}$ Tra i tanti possibili riferimenti, v. di recente A. Spadaro, L'evoluzione della forma di governo, cit., p. 89; S. Cassese, Con il governo è cambiata anche la forma di governo?, cit., pp. 116; A. Ruggeri, Evoluzione del sistema politico- istituzionale e ruolo del Presidente della Repubblica (Relazione introduttiva al Convegno dall'omonimo titolo svoltosi a Messina e Siracusa il 19 - 20 novembre 2010 ), in «Forum di Quaderni Costituzionali» (www.forumcostituzionale.it), 20/11/2010, pp. 6 ss., ed in Evoluzione del sistema politico- istituzionale e ruolo del Presidente della Repubblica, a cura di A. Ruggeri, cit., pp. 3 ss. (il quale sottopone peraltro a critica questa formula dottrinaria tradizionale). In precedenza, v. A. Di Giovine, Dieci anni di presidenza della Repubblica, in Il Presidente della Repubblica, a cura di M. Luciani e M. Volpi, Bologna, Il Mulino, 1997, pp. 25 ss..

${ }^{107}$ Per esempio, Lorenza Carlassare, Gianni Ferrara e Gaetano Azzariti (le cui più recenti riflessioni sulla problematica in esame sono reperibili nella rivista «Costituzionalismo.it» - www. costituzionalismo.it); ma v. già in precedenza, per l'utilizzo dell'espressione «presidenzialismo 
strisciante» in riferimento all'evoluzione della forma di governo in Italia, M. Volpi, Il Presidente della Repubblica tra presidenzialismo strisciante e presidenzialismo impotente, in Il Presidente della Repubblica, a cura di M. Luciani e M. Volpi, cit., pp. 459 s..

${ }^{108}$ Sull'evoluzione del ruolo e della figura istituzionale del presidente della repubblica - in relazione ai problemi ed alle prospettive future della forma di governo in Italia - v. in particolare, di recente, i volumi Evoluzione del sistema politico-istituzionale e ruolo del Presidente della Repubblica, a cura di A. Ruggeri, cit., e Il Presidente della Repubblica nell'evoluzione della forma di governo, a cura di A. Baldassarre e G. Scaccia, Roma, Aracne, 2012 (che raccolgono gli atti dei due omonimi Convegni svoltisi, rispettivamente, a Messina e Siracusa il 19 - 20 novembre 2010 e a Roma, Università "Luiss Guido Carli" il 26 novembre 2010); M. Luciani, La gabbia del Presidente, in «Rivista A.I.C.» (www.rivistaaic.it), 2013, n. 2; Id., parabola della Presidenza della Repubblica, in «Rivista A.I.C.» (www.rivistaaic.it), 2014, n. 1; S. Sicardi, Stranezze costituzionali? I tormentati esordi (a dir poco) della XVII Legislatura repubblicana, cit., § 4.2.

${ }^{109} \mathrm{Si}$ tratta infatti, evidentemente, di un aspetto appare particolarmente delicato e controverso, in quanto tocca l'essenza stessa del ruolo e della figura del presidente della repubblica nell'ordinamento costituzionale italiano.

${ }^{110}$ Salvo forse il presidente Mattarella, eletto nel 2015 ed il cui mandato (di sette anni) è perciò tuttora in corso.

${ }^{111}$ Relazione pubblicata sul sito Internet della presidenza della repubblica (http://presidenti. quirinale.it/Napolitano/qrnw/statico/attivita/consultazioni/c_20mar2013/gruppi_lavoro/2013-04-12_relazione_finale.pdf), insieme alla Relazione finale dell'altro Gruppo di Lavoro ristretto nominato contestualmente dal presidente Napolitano, sulle «riforme economico-sociali». Il mandato del Gruppo di Lavoro sulle «riforme istituzionali», citato nel testo, è indicato espressamente a p. 4 della Relazione finale.

112 Il testo integrale del discorso in questione, tenuto nella seduta comune delle camere del 22 aprile 2013, è pubblicato, in particolare, sul sito Internet della presidenza della repubblica (http:// presidenti.quirinale.it/elementi/Continua.aspx?tipo=Discorso\&key=2688).

${ }^{113}$ Da segnalare, al riguardo, la posizione recentemente sostenuta da S. Cassese, Con il governo è cambiata anche la forma di governo?, cit., p. 116, secondo cui il presidente della Repubblica svolgerebbe «un ruolo attivo in campo politico, ben diverso da quello di garante che gli viene assegnato dalla retorica costituzionalistica».

${ }^{114} \mathrm{Cfr}$. in particolare, da ultimo, A. Spadaro, L'evoluzione della forma di governo, cit., spec. pp. $81 \mathrm{ss}$. e 96 ss., che ha definito la forma di governo italiana «parlamentare con supplenza degli organi di garanzia» e - più specificamente - «con moderati tratti presidenziali».

115 Nell'ambito del noto processo sulla c.d. «trattativa Stato - mafia». Per la ricostruzione della vicenda processuale e delle argomentazioni della corte, nella già ampia bibliografia formatasi, si vedano i contributi pubblicati nel sito «Consulta On Line» a cura di Pasquale Costanzo, al link relativo a tale pronuncia (http://www.giurcost.org/decisioni/index.html).

116 Sentenza n. 1/2013, punto 8 del «Considerato in diritto».

${ }^{117}$ In Forme di Stato e forme di governo, cit., p. 2603.

118 In Le forme di governo, cit., p. 705.

$119 \mathrm{~V}$. più ampiamente oltre, $\S 5$.

${ }^{120}$ V. retro, $§ 2.1$.

${ }^{121}$ Questa tendenza "rovescia" lo schema dei rapporti tra i partiti tipico del sistema elettorale proporzionale, nel quale le alleanze politiche vengono fatte dopo le elezioni, e rende possibile nel periodo considerato la strutturazione del sistema politico italiano (sia pure in modo molto graduale e "faticoso") in senso sostanzialmente «bipolare», con anche una diminuzione del numero dei gruppi parlamentari.

${ }^{122}$ La c.d. «presidenzializzazione» dell'Esecutivo si ritrova, peraltro, anche in altre coeve esperienze costituzionali: v. A. Di Giovine e A. Mastromarino, La presidenzializzazione degli esecutivi nelle democrazie contemporanee, Torino, Giappichelli, 2007.

${ }^{123}$ Come si è visto nel $§ 2.1$, senza più partiti cc.dd. «antisistema» ed esclusi "a priori" dalla partecipazione alla maggioranza di governo (come avveniva invece prima del 1993). 
${ }^{124} \mathrm{~V}$. al riguardo sopra, $\S \S 2.1$ - 2.2. Ciò comporta - come nel periodo prima del 1993 - la rottura delle coalizioni di governo durante le legislature e, di conseguenza, la precarietà ed instabilità dei governi.

${ }^{125}$ Sempre nel $\S 2.2$, e poi 3.

${ }^{126}$ I quali anzi hanno continuato ad operare nel sistema politico come in precedenza, in modo conflittuale e "fluido".

${ }^{127}$ Forse è possibile affermare - ad uno sguardo retrospettivo sull'intero venticinquennio qui esaminato (1993/2018) - che in Italia vi è stata una "transizione incompiuta" dal parlamentarismo «compromissorio» al «maggioritario».

${ }^{128}$ In un contesto di bicameralismo «paritario» (o «perfetto»), in cui la fiducia viene conferita al governo da entrambe le camere.

${ }^{129} \mathrm{Al}$ combinato disposto del primo e del quarto comma dell'art. 94 della Carta.

${ }^{130}$ Espressa nel famoso «ordine del giorno Perassi», appunto sulla forma di governo parlamentare.

${ }^{131}$ Se ne accennerà nel paragrafo seguente.

${ }^{132}$ Sistema in vigore, con alcune modifiche (in particolare, legge n. 270/2005), fino alle sentenze n. $1 / 2014$ e n. 35/2017 della corte costituzionale ed alla legge n. 165/2017, già ricordate.

${ }^{133}$ Sottolineano questa «continuità formale» del testo costituzionale, per esempio, N. Lupo e G. Piccirilli, Le recenti evoluzioni della forma di governo italiana: una conferma della sua natura parlamentare, in www.astrid-online.it ed in «Democrazia e diritto», 2012, pp. 85 ss.

${ }^{134}$ Per questa definizione v. la sentenza n. 1/2013 della corte costituzionale (richiamata nel § 3).

${ }^{135}$ Esse sono state, peraltro, già in gran parte ricordate nei paragrafi precedenti (in particolare, $\S \S$ 2.1 - 2.5), nel contesto dell'analisi dei diversi aspetti concreti della forma di governo italiana.

${ }^{136} \mathrm{Fa}$ eccezione solo, forse, il caso dello scioglimento decretato nel gennaio del 1994 dal presidente Scalfaro, al quale si è fatto riferimento nel § 2.3.

${ }^{137} \mathrm{Nel}$ quadro di una rinnovata - ma assai discutibile e preoccupante, dal punto di vista del diritto costituzionale - concezione del potere costituente e del suo esercizio basata su un rapporto sostanzialmente "plebiscitario» tra gli organi politici di vertice ed il popolo/corpo elettorale (ampi riferimenti su questa problematica in S. Sicardi, Costituzione, potere costituente e revisione costituzionale, in Vent'anni di Costituzione, cit., pp. 46 ss.).

${ }^{138} \mathrm{Cfr}$. oltre, $\S 6$ (e relativi riferimenti).

${ }^{139}$ Riferimenti a questa argomentazione (talvolta, peraltro, forse fin troppo critici), per esempio, in L. Carlassare, Maggioritario, in «Costituzionalismo.it» (www.costituzionalismo.it), 2008, n. 1, ed in P. Caretti, Principio maggioritario e democraticità del sistema costituzionale, ibidem (il quale osserva che, negli anni a noi più vicini, il «principio maggioritario ... in sé» è stato reputato «una sorta di panacea di ogni male»).

140 V. sopra, § 4 .

${ }^{141}$ Rileva il ritorno della «centralità» del Parlamento nell'attuale contesto, per es., M. Ainis, La forma di governo della Terza Repubblica, cit., p. 113.

${ }^{142}$ Tra i tanti possibili riferimenti in proposito (per i quali si rinvia alla bibliografia ragionata in calce al capitolo), si segnalano R. Cherchi, La forma di governo, cit., § 3 (che rileva, in particolare, che l'Assemblea costituente «diede ... attuazione all'ordine del giorno Perassi in modo parziale», dato che le disposizioni costituzionali relative ai «più significativi rapporti politici - segnatamente, [alla] definizione dei rapporti endogovernativi, i poteri del Governo in Parlamento, il potere di scioglimento delle Camere ... erano ... così elastiche, pur se non completamente indefinite, da lasciare al dopo la specificazione del loro ubi consistam»); A. Ruggeri, Le crisi di governo tra "regole" costituzionali e "regolarità" della politica, cit., pp. 40 ss. (il quale osserva che «il modello costituzionale ... [presenta] una formidabile "apertura" strutturale, che lo rende disponibile a farsi variamente "impressionare" e sostanzialmente "riplasmare" dalle più marcate "regolarità"»); M. Timiani, Crisi e formazione dei governi, cit., p. 69 (che afferma espressamente che «il modello di parlamentarismo consensuale che si impose nel corso della c.d. "Prima Repubblica" ... fu ... solo "una delle possibili modalità di realizzazione" del dettato costituzionale; come "una delle possibili modalità di realizzazione" è "la democrazia maggioritaria" ... la quale "si colloca legittimamente all'interno della Costituzione"»). 
${ }^{143}$ Su cui si veda S. Sicardi, M. Cavino, L. Conte, L. Imarisio, G. Sobrino, C. Tripodina, La riforma respinta (2014 - 2016). Riflessioni sul d.d.l. costituzionale Renzi - Boschi, Bologna, Il Mulino, 2017. Va ricordato che nella legislatura attualmente in corso (iniziata nel 2018), e precisamente a seguito di un referendum ex art. 138 Cost. svoltosi il 20-21 settembre 2020, è stata approvata una revisione costituzionale consistente nella riduzione (populisticamente definita, in particolare dal MoVimento 5 Stelle che per primo l'ha proposta, "taglio") del numero dei membri delle Camere: da 630 a 400 alla Camera dei deputati e da 315 a 200 al Senato della Repubblica. Si tratta peraltro di una riforma costituzionale che, sebbene non del tutto priva di possibili riflessi sulla forma di governo, non la investe direttamente, né tocca - in particolare - i rapporti tra il parlamento ed il governo.

144 Spesso però legate all'instabilità delle maggioranze politiche che le hanno elaborate (un altro elemento caratterizzante il periodo successivo alla "svolta" del 1993 - sul quale non è possibile qui svolgere le opportune riflessioni - è invero, la circostanza che le riforme della Costituzione sono state proposte di norma dalle maggioranze politiche di volta in volta prevalenti, e non condivise tra la maggioranza e l'opposizione).

145 Sia consentito rinviare a G. Sobrino, Il percorso delle riforme costituzionali, in S. Sicardi, M. Cavino, L. Conte, L. Imarisio, G. Sobrino, C. Tripodina, La riforma respinta, cit., pp. 15 ss.

146 Il testo del progetto in questione (così come dei progetti riportati di seguito nel testo) è consultabile, in particolare, sul sito Internet della camera dei deputati, al link http://www.camera.it/ parlam/bicam/rifcost/dossier/prec07.htm.

147 Testo consultabile al link http://www.camera.it/parlam/bicam/rifcost/dossier/prec08.htm.

${ }^{148}$ V., al riguardo, i materiali al link http://www.camera.it/parlam/bicam/rifcost/, nonché $L a$ Commissione Bicamerale per le Riforme Costituzionali. I progetti, i lavori, i testi approvati, a cura di P. Costanzo, G.F. Ferrari, G.G. Floridia, R. Romboli e S. Sicardi, Padova, Cedam, 1998.

${ }^{149}$ Consultabile (in utile comparazione sinottica con quelli dei progetti che lo hanno preceduto, nonché con quello approvato dal senato nella legislatura successiva - la XVI - nel 2012, A.C. n. 5386) nel Dossier del Servizio studi del senato, maggio 2013, n. 14, reperibile al link http:// www.senato.it/service/PDF/PDFServer/BGT/00739725.pdf.

${ }^{150}$ Reperibile sul sito Internet della presidenza della repubblica (http://presidenti.quirinale.it/ Napolitano/qrnw/statico/attivita/consultazioni/c_20mar2013/gruppi_lavoro/2013-04-12_relazione_finale.pdf).

${ }^{151}$ Reperibile sul sito Internet del ministero per le Riforme costituzionali (al link http://riformecostituzionali.gov.it/commissione-riforme.html).

152 Su cui si v. ancora S. Sicardi, M. Cavino, L. Conte, L. Imarisio, G. Sobrino, C. Tripodina, La riforma respinta, cit.

Recebido em: 30-11-2020

Autor convidado

\title{
Giorgio Sobrino
}

Ricercatore di Diritto Costituzionale, Università degli Studi di Torino. E-mail: giorgio.sobrino@unito.it

\author{
Università degli Studi di Torino, Dipartimento di Giurisprudenza, Campus Luigi \\ Einaudi, Lungo Dora Siena 100/A, 10153 - Torino.
}

